\title{
A Finite-Volume Method for Nonlinear Nonlocal Equations with a Gradient Flow Structure
}

\author{
José A. Carrillo, Alina Chertock†, and Yanghong Huang $\ddagger$
}

August 4, 2014

\begin{abstract}
We propose a positivity preserving entropy decreasing finite volume scheme for nonlinear nonlocal equations with a gradient flow structure. These properties allow for accurate computations of stationary states and long-time asymptotics demonstrated by suitably chosen test cases in which these features of the scheme are essential. The proposed scheme is able to cope with non-smooth stationary states, different time scales including metastability, as well as concentrations and self-similar behavior induced by singular nonlocal kernels. We use the scheme to explore properties of these equations beyond their present theoretical knowledge.
\end{abstract}

\section{Introduction}

In this paper, we consider a finite-volume method for the following problem:

$$
\left\{\begin{array}{l}
\rho_{t}=\nabla \cdot\left[\rho \nabla\left(H^{\prime}(\rho)+V(\mathbf{x})+W * \rho\right)\right], \quad \mathbf{x} \in \mathbb{R}^{d}, t>0 \\
\rho(\mathbf{x}, 0)=\rho_{0}(\mathbf{x}),
\end{array}\right.
$$

where $\rho(\mathbf{x}, t) \geq 0$ is the unknown probability measure, $W(\mathbf{x})$ is an interaction potential, which is assumed to be symmetric, $H(\rho)$ is a density of internal energy, and $V(\mathbf{x})$ is a confinement potential.

Equations such as (1.1) appear in various contexts. If $W$ and $V$ vanishes, and $H(\rho)=$ $\rho \log \rho-\rho$ or $H(\rho)=\rho^{m}$, it is the classical heat equation or porous medium/fast diffusion equation [38]. If mass-conserving, self-similar solutions of these equations are sought, the quadratic term $V(\mathbf{x})=|\mathbf{x}|^{2}$ is added, leading to new equations in similarity variables. More generally, $V$ usually appears as a confining potential in Fokker-Planck type equations [19,31]. Finally, $W$ is related to the interaction energy, and can be as singular as the Newtonian potential in chemotaxis system [25] or as smooth as $W(\mathbf{x})=|\mathbf{x}|^{\alpha}$ with $\alpha>2$ in granular flow [4].

\footnotetext{
*Department of Mathematics, Imperial College London, London SW7 2AZ, UK; carrillo@imperial.ac.uk

†Department of Mathematics, North Carolina State University, Raleigh, NC 27695, USA; chertock@math.ncsu.edu

${ }_{\ddagger}^{\ddagger}$ Department of Mathematics, Imperial College London, London SW7 2AZ, UK; yanghong. huang@imperial.ac.uk
} 
The free energy associated to equation (1.1) is given by (see $[17,18,40])$ :

$$
E(\rho)=\int_{\mathbb{R}^{d}} H(\rho) d \mathbf{x}+\int_{\mathbb{R}^{d}} V(\mathbf{x}) \rho(\mathbf{x}) d \mathbf{x}+\frac{1}{2} \int_{\mathbb{R}^{d}} \int_{\mathbb{R}^{d}} W(\mathbf{x}-\mathbf{y}) \rho(\mathbf{x}) \rho(\mathbf{y}) d \mathbf{x} d \mathbf{y} .
$$

This energy functional is the sum of internal energy, potential energy and interaction energy, corresponding to the three terms on the right-hand side of (1.2), respectively. A simple computation shows that, at least for classical solutions, the time-derivative of $E(\rho)$ along solutions of $(1.1)$ is

$$
\frac{d}{d t} E(\rho)=-\int_{\mathbb{R}^{d}} \rho|\mathbf{u}|^{2} d \mathbf{x}:=-I(\rho)
$$

where

$$
\mathbf{u}=-\nabla \xi, \quad \xi:=\frac{\delta E}{\delta \rho}=H^{\prime}(\rho)+V(\mathbf{x})+W * \rho .
$$

The functional $I$ will henceforth be referred to as the entropy dissipation functional.

The equation (1.1) and its associated energy $E(\rho)$ are the subjects of intensive study during the past fifteen years, see e.g. $[1,17,29,40]$ and the references therein. The general properties of (1.1) are investigated in the context of interacting gases [17,29,40], and are common to a wide variety of models, including granular flows $[3,4,27,36]$, porous medium flows $[19,31]$, and collective behavior in biology [35]. The gradient flow structure, in the sense of (1.3), is generalized from smooth solutions to measure-valued solutions [1]. Certain entropy-entropy dissipation inequalities between $E(\rho)$ and $I(\rho)$ are also recognized to characterize the fine details of the convergence to steady states $[17,19,31]$.

The steady state of (1.1), if it exists, usually verifies the form

$$
\xi=H^{\prime}(\rho)+V(\mathbf{x})+W * \rho=C, \quad \text { on } \operatorname{supp} \rho,
$$

where the constant $C$ could be different on different connected components of supp $\rho$. In many cases, especially in the presence of the interaction potential $W$, there are multiple steady states, whose explicit forms are available only for particular $W$. Most of studies of these steady states are based on certain assumptions on the support and the characterizing equation (1.5).

In this work, we propose a positivity preserving finite-volume method to treat the general nonlocal nonlinear PDE (1.1). Moreover, we show the existence of a discrete free energy that is dissipated for the semi-discrete scheme (discrete in space only). A related method was already proposed in [5] for the case of nonlinear degenerate diffusions in any dimension. We generalize this method to cover the nonlocal terms for both 1D and 2D cases in Section 2. In fact, the first order scheme generalizes easily to cover unstructured meshes. However, it is an open problem how to obtain entropy decreasing higher order schemes in this setting in 2D. Let us remark that other numerical methods based on finite element approximations have been proposed in the literature which are positivity preserving and entropy decreasing at the expense of constructing them by an implicit discretization in time but continuous in space, see [9].

Section 3 is devoted to numerical experiments, in which the performance of the developed numerical approach is tested. In Section 3.1, we conduct the convergence study of stationary states, where the order of accuracy depends on the regularity at free boundaries. We then showcase the performance of this method for finding stable stationary states with nonlocal terms and their equilibration rate in time for different nonlocal models. In Section 3.2, we emphasize how this method is useful to explore different open problems in the analysis of these 
nonlocal nonlinear models such as the Keller-Segel model for chemotaxis in its different versions. We continue in Section 3.3 with aggregation equations with repulsive-attractive kernels and address the issue of singular kernels and discontinuous steady states. Finally, in Section 3.4, we demonstrate the performance of the scheme in a number of $2-\mathrm{D}$ experiments showcasing numerical difficulties and interesting asymptotics.

\section{Numerical Method}

In this section, we describe both one- (1-D) and two-dimensional (2-D) finite-volume schemes for (1.1) and prove their positivity preserving and entropy dissipation properties. We also establish error estimates and convergence results for the proposed methods. We start in $\S 2.1$ with the $1-\mathrm{D}$ case and then generalize it to the $2-\mathrm{D}$ case in $\S 2.2$, both on uniform meshes. The extension to higher dimensions and non-uniform structured meshes is straightforward.

\subsection{One-Dimensional Case}

We begin with the derivation of the 1-D second-order finite-volume method for equation (1.1). For simplicity, we divide the computational domain into finite-volume cells $C_{j}=\left[x_{j-\frac{1}{2}}, x_{j+\frac{1}{2}}\right]$ of a uniform size $\Delta x$ with $x_{j}=j \Delta x, j \in\{-M, \cdots, M\}$, and denote by

$$
\bar{\rho}_{j}(t)=\frac{1}{\Delta x} \int_{C_{j}} \rho(x, t) d x,
$$

the computed cell averages of the solution $\rho$, which we assume to be known or approximated at time $t \geq 0$. A semi-discrete finite-volume scheme is obtained by integrating equation (1.1) over each cell $C_{j}$ and is given by the following system of ODEs for $\bar{\rho}_{j}$ :

$$
\frac{d \bar{\rho}_{j}(t)}{d t}=-\frac{F_{j+\frac{1}{2}}(t)-F_{j-\frac{1}{2}}(t)}{\Delta x},
$$

where the numerical flux $F_{j+\frac{1}{2}}$ approximate the continuous flux $-\rho \xi_{x}=-\rho\left(H^{\prime}(\rho)+V(x)+W *\right.$

$\rho)_{x}$ at cell interface $x_{j+\frac{1}{2}}$ and is constructed next. For simplicity, we will omit the dependence of the computed quantities on $t \geq 0$ in the rest. As in the case of degenerate diffusion equations treated in [5], we use the upwind numerical fluxes. To this end, we first construct piecewise linear polynomials in each cell $C_{j}$,

$$
\widetilde{\rho}_{j}(x)=\bar{\rho}_{j}+\left(\rho_{x}\right)_{j}\left(x-x_{j}\right), \quad x \in C_{j},
$$

and compute the right ("east"), $\rho_{j}^{\mathrm{E}}$, and left ("west"), $\rho_{j}^{\mathrm{W}}$, point values at the cell interfaces $x_{j-\frac{1}{2}}$ and $x_{j+\frac{1}{2}}$, respectively:

$$
\begin{aligned}
& \rho_{j}^{\mathrm{E}}=\widetilde{\rho}_{j}\left(x_{j+\frac{1}{2}}-0\right)=\bar{\rho}_{j}+\frac{\Delta x}{2}\left(\rho_{x}\right)_{j}, \\
& \rho_{j}^{\mathrm{W}}=\widetilde{\rho}_{j}\left(x_{j-\frac{1}{2}}+0\right)=\bar{\rho}_{j}-\frac{\Delta x}{2}\left(\rho_{x}\right)_{j} .
\end{aligned}
$$

These values will be second-order accurate provided the numerical derivatives $\left(\rho_{x}\right)_{j}$ are at least first-order accurate approximations of $\rho_{x}(x, \cdot)$. To ensure that the point values $(2.3)$ are both 
second-order and nonnegative, the slopes $\left(\rho_{x}\right)_{j}$ in $(2.2)$ are calculated according to the following adaptive procedure. First, the centered-difference approximations $\left(\rho_{x}\right)_{j}=\left(\bar{\rho}_{j+1}-\bar{\rho}_{j-1}\right) /(2 \Delta x)$ is used for all $j$. Then, if the reconstructed point values in some cell $C_{j}$ become negative (i.e., either $\rho_{j}^{\mathrm{E}}<0$ or $\rho_{j}^{\mathrm{W}}<0$ ), we recalculate the corresponding slope $\left(\rho_{x}\right)_{j}$ using a slope limiter, which guarantees that the reconstructed point values are nonnegative as long as the cell averages $\bar{\rho}_{j}$ are nonnegative. In our numerical experiments, we have used a generalized minmod limiter $[28,30,34,37]$ :

$$
\left(\rho_{x}\right)_{j}=\operatorname{minmod}\left(\theta \frac{\bar{\rho}_{j+1}-\bar{\rho}_{j}}{\Delta x}, \frac{\bar{\rho}_{j+1}-\bar{\rho}_{j-1}}{2 \Delta x}, \theta \frac{\bar{\rho}_{j}-\bar{\rho}_{j-1}}{\Delta x}\right),
$$

where

$$
\operatorname{minmod}\left(z_{1}, z_{2}, \ldots\right):= \begin{cases}\min \left(z_{1}, z_{2}, \ldots\right), & \text { if } z_{i}>0 \forall i \\ \max \left(z_{1}, z_{2}, \ldots\right), & \text { if } z_{i}<0 \quad \forall i \\ 0, & \text { otherwise }\end{cases}
$$

and the parameter $\theta$ can be used to control the amount of numerical viscosity present in the resulting scheme. In all the numerical examples below, $\theta=2$ is used.

Equipped with the piecewise linear reconstruction $\widetilde{\rho}_{j}(x)$ and point values $\rho_{j}^{\mathrm{E}}, \rho_{j}^{\mathrm{W}}$, the upwind fluxes in (2.1) are computed as

$$
F_{j+\frac{1}{2}}=u_{j+\frac{1}{2}}^{+} \rho_{j}^{\mathrm{E}}+u_{j+\frac{1}{2}}^{-} \rho_{j+1}^{\mathrm{W}},
$$

where the discrete values $u_{j+\frac{1}{2}}$ of the velocities are obtained using the centered-difference approach,

$$
u_{j+\frac{1}{2}}=-\frac{\xi_{j+1}-\xi_{j}}{\Delta x}
$$

and the positive and negative parts of $u_{j+\frac{1}{2}}$ are denoted by

$$
u_{j+\frac{1}{2}}^{+}=\max \left(u_{j+\frac{1}{2}}, 0\right), \quad u_{j+\frac{1}{2}}^{-}=\min \left(u_{j+\frac{1}{2}}, 0\right) .
$$

The discrete velocity field $\xi_{j}$ is calculated by discretizing (1.4):

$$
\xi_{j}=\Delta x \sum_{i} W_{j-i} \bar{\rho}_{i}+H^{\prime}\left(\bar{\rho}_{j}\right)+V_{j}
$$

where $W_{j-i}=W\left(x_{j}-x_{i}\right)$ and $V_{j}=V\left(x_{j}\right)$. The formula (2.7) is a second-order approximation of

$$
\sum_{i} \int_{C_{i}} W\left(x_{j}-s\right) \widetilde{\rho}_{i}(s) d s+H^{\prime}\left(\widetilde{\rho}_{j}\left(x_{j}\right)\right)+V\left(x_{j}\right) .
$$

Indeed, the reconstruction $(2.2)$ yields $H^{\prime}\left(\widetilde{\rho}_{j}\left(x_{j}\right)\right)=H^{\prime}\left(\bar{\rho}_{j}\right)$ and

$$
\begin{aligned}
\sum_{i} \int_{C_{i}} W\left(x_{j}-s\right) \widetilde{\rho}_{i}(s) d s & =\sum_{i} \bar{\rho}_{i} \int_{C_{i}} W\left(x_{j}-s\right) d s+\sum_{i}\left(\rho_{x}\right)_{i} \int_{C_{i}} W\left(x_{j}-s\right)\left(s-x_{i}\right) d s \\
& =\Delta x \sum_{i} W_{j-i} \bar{\rho}_{i}+\mathcal{O}\left(\Delta x^{2}\right)
\end{aligned}
$$


Here $W_{j-i}$ can be any approximation of the local integral $\frac{1}{\Delta x} \int_{C_{i}} W\left(x_{j}-s\right) d s$ with error $O\left(\Delta x^{2}\right)$. If $W$ has a bounded second order derivative near $x_{j-i}, W_{j-i}$ can be chosen to be $W\left(x_{j-i}\right)$ (the middle point rule) or $\left(W\left(x_{j-i-1 / 2}\right)+W\left(x_{j-i+1 / 2}\right)\right) / 2$ (the trapezoidal rule). The integral $\int_{C_{i}} W\left(x_{j}-s\right)\left(s-x_{i}\right) d s$ in the second summation is of $O\left(\Delta x^{3}\right)$ because of the anti-symmetric factor $s-x_{i}$, leading to overall error $O\left(\Delta x^{2}\right)$.

The case with non-smooth or singular interaction potential $W$ has to be treated more carefully. First, the last integral $\int_{C_{i}} W\left(x_{j}-s\right)\left(s-x_{i}\right) d s$ in the above formula vanishes as soon as $i=j$ due to the symmetry of $W$ independently of any possible singularity at $x=x_{j}$. If $W$ has a locally integrable singularity (usually at the origin), $\frac{1}{\Delta x} \int_{C_{i}} W\left(x_{j}-s\right) d s$ can still be approximated by a higher order quadrature scheme with an error $O\left(\Delta x^{2}\right)$ or smaller. Actually, in the particular case of powers or logarithm kernels, it can be explicitly computed. However, the second sum above may have a slightly larger error. For instance, if $W(x) \sim|x|^{-\alpha}$ for $0<\alpha<1$, then $\int_{C_{i}} W\left(x_{j}-s\right)\left(s-x_{i}\right) d s \sim O\left(\Delta x^{2-\alpha}\right)$ by direct computation when $|i-j|$ is close to zero.

Finally, the semi-discrete scheme (2.1) is a system of ODEs, which has to be integrated numerically using a stable and accurate ODE solver. In all numerical examples reported in next section, the third-order strong preserving Runge-Kutta (SSP-RK) ODE solver [24] is used.

Remark 2.1. The computational bottleneck is the discrete convolution in (2.7). This is a classical problem in scientific computing that can be effectively evaluated using fast convolution algorithms, mainly based Fast Fourier Transforms [41].

Remark 2.2. The second-order finite-volume scheme (2.1), (2.4)-(2.7), reduces to the first-order one if the piecewise constant reconstruction is used instead of (2.2), in which case one has

$$
\widetilde{\rho}_{j}(x)=\bar{\rho}_{j}, \quad x_{j} \in C_{j}, \quad \text { and therefore } \quad \rho_{j}^{\mathrm{E}}=\rho_{j}^{\mathrm{W}}=\bar{\rho}_{j}, \quad \forall j .
$$

Positivity Preserving. The resulting scheme preserves positivity of the computed cell averages $\bar{\rho}_{j}$ as stated in the following theorem. The proof is based on the forward Euler integration of the ODE system (2.1), but will remain equally valid if the forward Euler method were replaced by a higher-order SSP ODE solver [24], whose time step can be expressed as a convex combination of several forward Euler steps.

Theorem 2.3. Consider the system (1.1) with initial data $\rho_{0}(x) \geq 0$ and the semi-discrete finite-volume scheme (2.1), (2.4)-(2.7) with a positivity preserving piecewise linear reconstruction (2.2) for $\rho$. Assume that the system of ODEs (2.1) is discretized by the forward Euler method. Then, the computed cell averages $\bar{\rho}_{j} \geq 0, \forall j$, provided that the following CFL condition is satisfied:

$$
\Delta t \leq \frac{\Delta x}{2 a}, \quad \text { where } a=\max _{j}\left\{u_{j+\frac{1}{2}}^{+},-u_{j+\frac{1}{2}}^{-}\right\}
$$

with $u_{j+\frac{1}{2}}^{+}$and $u_{j+\frac{1}{2}}^{-}$defined in (2.6).

Proof. Assume that at a given time $t$ the computed solution is known and positive: $\bar{\rho}_{j} \geq 0, \forall j$. Then the new cell averages are obtained from the forward Euler discretization of equation (2.1):

$$
\bar{\rho}_{j}(t+\Delta t)=\bar{\rho}_{j}(t)-\lambda\left[F_{j+\frac{1}{2}}(t)-F_{j-\frac{1}{2}}(t)\right]
$$


where $\lambda:=\Delta t / \Delta x$. As above, the dependence of all terms on the RHS of (2.10) on $t$ is suppressed in the following to simplify the notation. Using (2.4) and the fact that $\bar{\rho}_{j}=\frac{1}{2}\left(\rho_{j}^{\mathrm{E}}+\rho_{j}^{\mathrm{W}}\right)$ (see $(2.3)$ ), we obtain

$$
\begin{aligned}
\bar{\rho}_{j}(t+\Delta t) & =\frac{1}{2}\left(\rho_{j}^{\mathrm{E}}+\rho_{j}^{\mathrm{W}}\right)-\lambda\left[u_{j+\frac{1}{2}}^{+} \rho_{j}^{\mathrm{E}}+u_{j+\frac{1}{2}}^{-} \rho_{j+1}^{\mathrm{W}}-u_{j-\frac{1}{2}}^{+} \rho_{j-1}^{\mathrm{E}}-u_{j-\frac{1}{2}}^{-} \rho_{j}^{\mathrm{W}}\right] \\
& =\lambda u_{j-\frac{1}{2}}^{+} \rho_{j-1}^{\mathrm{E}}+\left(\frac{1}{2}-\lambda u_{j+\frac{1}{2}}^{+}\right) \rho_{j}^{\mathrm{E}}+\left(\frac{1}{2}+\lambda u_{j-\frac{1}{2}}^{-}\right) \rho_{j}^{\mathrm{W}}-\lambda u_{j+\frac{1}{2}}^{-} \rho_{j+1}^{\mathrm{W}} .
\end{aligned}
$$

It follows from (2.11) that the new cell averages $\bar{\rho}_{j}(t+\Delta t)$ are linear combinations of the nonnegative reconstructed point values $\rho_{j-1}^{\mathrm{E}}, \rho_{j}^{\mathrm{E}}, \rho_{j}^{\mathrm{W}}$ and $\rho_{j+1}^{\mathrm{W}}$. Since $u_{j-\frac{1}{2}}^{+} \geq 0$ and $u_{j+\frac{1}{2}}^{-} \leq 0$, we conclude that $\bar{\rho}_{j}(t+\Delta t) \geq 0, \forall j$, provided that the CFL condition (2.9) is satisfied.

Remark 2.4. Similar result holds for the first-order finite-volume scheme with the CFL condition reduced to

$$
\Delta t \leq \frac{\Delta x}{2 \max _{j}\left(u_{j+\frac{1}{2}}^{+}-u_{j-\frac{1}{2}}^{-}\right)}
$$

Discrete Entropy Dissipation. A discrete version of the entropy $E$ defined in (1.2) is given by

$$
E_{\Delta}(t)=\Delta x \sum_{j}\left[\frac{1}{2} \Delta x \sum_{i} W_{j-i} \bar{\rho}_{i} \bar{\rho}_{j}+H\left(\bar{\rho}_{j}\right)+V_{j} \bar{\rho}_{j}\right] .
$$

We also introduce the discrete version of the entropy dissipation

$$
I_{\Delta}(t)=\Delta x \sum_{j}\left(u_{j+\frac{1}{2}}\right)^{2} \min _{j}\left(\rho_{j}^{\mathrm{E}}, \rho_{j+1}^{\mathrm{W}}\right) .
$$

In the following theorem, we prove that the time derivative of $E_{\Delta}(t)$ is less or equal than the negative of $I_{\Delta}(t)$, mimicking the corresponding property of the continuous relation.

Theorem 2.5. Consider the system (1.1) with no flux boundary conditions on $[-L, L]$ with $L>0$ and with initial data $\rho_{0}(x) \geq 0$. Given the semi-discrete finite-volume scheme (2.1) with $\Delta x=L / M,(2.4)-(2.7)$ with a positivity preserving piecewise linear reconstruction (2.2) for $\rho$ and discrete boundary conditions $F_{M+\frac{1}{2}}=F_{-M-\frac{1}{2}}=0$. Then,

$$
\frac{d}{d t} E_{\Delta}(t) \leq-I_{\Delta}(t), \quad \forall t>0
$$

Proof. We start by differentiating (2.12) with respect to time to obtain:

$$
\begin{aligned}
\frac{d}{d t} E_{\Delta}(t) & =\Delta x \sum_{j}\left[\Delta x \sum_{i} W_{j-i} \bar{\rho}_{i} \frac{d \bar{\rho}_{j}}{d t}+H^{\prime}\left(\bar{\rho}_{j}\right) \frac{d \bar{\rho}_{j}}{d t}+V_{j} \frac{d \bar{\rho}_{j}}{d t}\right] \\
& =\Delta x \sum_{j}\left[\Delta x \sum_{i} W_{j-i} \bar{\rho}_{i}+H^{\prime}\left(\bar{\rho}_{j}\right)+V_{j}\right] \frac{d \bar{\rho}_{j}}{d t} .
\end{aligned}
$$


Using the definition (2.7) and the numerical scheme (2.1), we have

$$
\frac{d}{d t} E_{\Delta}(t)=-\Delta x \sum_{j} \xi_{j} \frac{F_{j+\frac{1}{2}}-F_{j-\frac{1}{2}}}{\Delta x}
$$

A discrete integration by parts using the no flux discrete boundary conditions along with (2.5) yields

$$
\frac{d}{d t} E_{\Delta}(t)=-\sum_{j}\left(\xi_{j}-\xi_{j+1}\right) F_{j+\frac{1}{2}}=-\Delta x \sum_{j} u_{j+\frac{1}{2}} F_{j+\frac{1}{2}} \cdot
$$

Finally, using the definition of the upwind fluxes (2.4) and formulas (2.6) and (2.13), we conclude

$$
\frac{d}{d t} E_{\Delta}(t)=-\Delta x \sum_{j} u_{j+\frac{1}{2}}\left[u_{j+\frac{1}{2}}^{+} \rho_{j}^{\mathrm{E}}+u_{j+\frac{1}{2}}^{-} \rho_{j+1}^{\mathrm{W}}\right] \leq-\Delta x \sum_{j}\left(u_{j+\frac{1}{2}}\right)^{2} \min _{j}\left(\rho_{j}^{\mathrm{E}}, \rho_{j+1}^{\mathrm{W}}\right)=-I_{\Delta}(t) .
$$

\subsection{Two-Dimensional Case}

In this subsection, we quickly describe a semi-discrete second-order finite-volume method for the 2-D equation (1.1). We explain the main ideas in 2D for the sake of the reader. As already mentioned, the first order scheme generalizes easily to unstructured meshes. However, higher order schemes with the desired entropy decreasing property are harder to obtain in this setting for higher dimensions. We introduce a Cartesian mesh consisting of the cells $C_{j, k}:=\left[x_{j-\frac{1}{2}}, x_{j+\frac{1}{2}}\right] \times\left[y_{k-\frac{1}{2}}, y_{k+\frac{1}{2}}\right]$, which for the sake of simplicity are assumed to be of the uniform size $\Delta x \Delta y$, that is, $x_{j+\frac{1}{2}}-x_{j-\frac{1}{2}} \equiv \Delta x, \forall j$, and $y_{k+\frac{1}{2}}-y_{k-\frac{1}{2}} \equiv \Delta y, \forall k$.

A general semi-discrete finite-volume scheme for equation (1.1) can be written in the following form:

$$
\frac{d \bar{\rho}_{j, k}}{d t}=-\frac{F_{j+\frac{1}{2}, k}^{x}-F_{j-\frac{1}{2}, k}^{x}}{\Delta x}-\frac{F_{j, k+\frac{1}{2}}^{y}-F_{j, k-\frac{1}{2}}^{y}}{\Delta y}
$$

Here, we define

$$
\bar{\rho}_{j, k}(t) \approx \frac{1}{\Delta x \Delta y} \iint_{C_{j, k}} \rho(x, y, t) d x d y
$$

as the cell averages of the computed solution and $F_{j+\frac{1}{2}, k}^{x}$ and $F_{j, k+\frac{1}{2}}^{y}$ are upwind numerical fluxes in the $x$ and $y$ directions, respectively.

As in the 1-D case, to obtain formulae for numerical fluxes, we first compute $\rho_{j, k}^{\mathrm{E}}, \rho_{j, k}^{\mathrm{W}}, \rho_{j, k}^{\mathrm{N}}$ and $\rho_{j, k}^{\mathrm{S}}$, which are one-sided point values of the piecewise linear reconstruction

$$
\widetilde{\rho}(x, y)=\bar{\rho}_{j, k}+\left(\rho_{x}\right)_{j, k}\left(x-x_{j}\right)+\left(\rho_{y}\right)_{j, k}\left(y-y_{k}\right), \quad(x, y) \in C_{j, k},
$$

at the cell interfaces $\left(x_{j+\frac{1}{2}}, y_{k}\right),\left(x_{j-\frac{1}{2}}, y_{k}\right),\left(x_{j}, y_{k+\frac{1}{2}}\right),\left(x_{j}, y_{k-\frac{1}{2}}\right)$, respectively. Namely,

$$
\begin{array}{ll}
\rho_{j, k}^{\mathrm{E}}:=\widetilde{\rho}\left(x_{j+\frac{1}{2}}-0, y_{k}\right)=\bar{\rho}_{j, k}+\frac{\Delta x}{2}\left(\rho_{x}\right)_{j, k}, \quad \rho_{j, k}^{\mathrm{W}}:=\widetilde{\rho}\left(x_{j-\frac{1}{2}}+0, y_{k}\right)=\bar{\rho}_{j, k}-\frac{\Delta x}{2}\left(\rho_{x}\right)_{j, k}, \\
\rho_{j, k}^{\mathrm{N}}:=\widetilde{\rho}\left(x_{j}, y_{k+\frac{1}{2}}-0\right)=\bar{\rho}_{j, k}+\frac{\Delta y}{2}\left(\rho_{y}\right)_{j, k}, \quad \rho_{j, k}^{\mathrm{S}}:=\widetilde{\rho}\left(x_{j}, y_{k-\frac{1}{2}}+0\right)=\bar{\rho}_{j, k}-\frac{\Delta y}{2}\left(\rho_{y}\right)_{j, k} .
\end{array}
$$


To ensure the point values in (2.16) are both second-order and nonnegative, the slopes in (2.15) are calculated according to the adaptive procedure similarly to the 1 -D case. First, the centered-difference approximations,

$$
\left(\rho_{x}\right)_{j, k}=\frac{\bar{\rho}_{j+1, k}-\bar{\rho}_{j-1, k}}{2 \Delta x} \quad \text { and } \quad\left(\rho_{y}\right)_{j, k}=\frac{\bar{\rho}_{j, k+1}-\bar{\rho}_{j, k-1}}{2 \Delta y}
$$

are used for all $j, k$. Then, if the reconstructed point values in some cell $C_{j, k}$ become negative, we recalculate the corresponding slopes $\left(\rho_{x}\right)_{j, k}$ or $\left(\rho_{y}\right)_{j, k}$ using a monotone nonlinear limiter, which guarantees that the reconstructed point values are nonnegative as long as the cell averages of $\bar{\rho}_{j, k}$ are nonnegative for all $j, k$. In our numerical experiments, we have used the one-parameter family of the generalized minmod limiters with $\theta \in[1,2]$ :

$$
\begin{gathered}
\left(\rho_{x}\right)_{j, k}=\operatorname{minmod}\left(\theta \frac{\bar{\rho}_{j, k}-\bar{\rho}_{j-1, k}}{\Delta x}, \frac{\bar{\rho}_{j+1, k}-\bar{\rho}_{j-1, k}}{2 \Delta x}, \theta \frac{\bar{\rho}_{j+1, k}-\bar{\rho}_{j, k}}{\Delta x}\right), \\
\left(\rho_{y}\right)_{j, k}=\operatorname{minmod}\left(\theta \frac{\bar{\rho}_{j, k}-\bar{\rho}_{j, k-1}}{\Delta y}, \frac{\bar{\rho}_{j, k+1}-\bar{\rho}_{j, k-1}}{2 \Delta y}, \theta \frac{\bar{\rho}_{j, k+1}-\bar{\rho}_{j, k}}{\Delta y}\right) .
\end{gathered}
$$

Given the polynomial reconstruction (2.15) and its point values (2.16), the upwind numerical fluxes in (2.14) are defined as

$$
F_{j+\frac{1}{2}, k}^{x}=u_{j+\frac{1}{2}, k}^{+} \rho_{j, k}^{\mathrm{E}}+u_{j+\frac{1}{2}, k}^{-} \rho_{j+1, k}^{\mathrm{W}}, \quad F_{j, k+\frac{1}{2}}^{y}=v_{j, k+\frac{1}{2}}^{+} \rho_{j, k}^{\mathrm{N}}+v_{j, k+\frac{1}{2}}^{-} \rho_{j, k+1}^{\mathrm{S}},
$$

where

$$
u_{j+\frac{1}{2}, k}=-\frac{\xi_{j+1, k}-\xi_{j, k}}{\Delta x}, \quad v_{j, k+\frac{1}{2}}=-\frac{\xi_{j, k+1}-\xi_{j, k}}{\Delta y},
$$

the values of $u_{j+\frac{1}{2}, k}^{ \pm}$and $v_{j, k+\frac{1}{2}}^{ \pm}$are defined according to (2.6), and

$$
\xi_{j, k}=\Delta x \Delta y \sum_{i, l} W_{j-i, k-l} \bar{\rho}_{i, l}+H^{\prime}\left(\bar{\rho}_{j, k}\right)+V_{j, k}
$$

Here, $W_{j-i, k-l}=W\left(x_{j}-x_{i}, y_{k}-y_{l}\right)$ and $V_{j, k}=V\left(x_{j}, y_{k}\right)$.

Similarly to the 1-D case, the formula (2.18) for $\xi_{j, k}$ is obtained by using the reconstruction formula (2.15) and applying the midpoint quadrature rule to the first integral in

$$
\xi_{j, k}=\sum_{i, l} \iint_{C_{i, l}} W(x-s, y-r) \widetilde{\rho}_{i, l}(s, r) d s d r+H^{\prime}\left(\widetilde{\rho}_{j, k}(x, y)\right)+V\left(x_{j}, y_{k}\right)
$$

As in the 1-D case, the ODE system (2.14) is to be integrated numerically by a stable and sufficiently accurate ODE solver such as the third-order SSP-RK ODE solver [24].

Remark 2.6. As in the 1-D case, the first-order finite-volume method is obtained by taking

$$
\tilde{\rho}_{j, k}(x, y)=\bar{\rho}_{j, k} \quad \text { and } \quad \rho_{j, k}^{\mathrm{E}}=\rho_{j, k}^{\mathrm{W}}=\rho_{j, k}^{\mathrm{N}}=\rho_{j, k}^{\mathrm{S}}=\bar{\rho}_{j, k}, \quad \forall j, k \text {. }
$$


Positivity Preserving. The resulting 2-D finite-volume scheme will preserve positivity of the computed cell averages $\bar{\rho}_{j, k}, \forall j, k$, as long as an SSP ODE solver, whose time steps are convex combinations of forward Euler steps, is used for time integration. We omit the proof of the positivity property of the scheme as it follows exactly the lines of Theorem 2.3. The only difference is that in the 2-D case $\bar{\rho}_{j, k}=\frac{1}{4}\left(\rho_{j, k}^{\mathrm{E}}+\rho_{j, k}^{\mathrm{W}}+\rho_{j, k}^{\mathrm{N}}+\rho_{j, k}^{\mathrm{S}}\right)$, which leads to a slightly modified CFL condition. We thus have the following theorem.

Theorem 2.7. Consider the system (1.1) with initial data $\rho_{0}(x) \geq 0$ and the semi-discrete finite-volume scheme (2.14), (2.17)-(2.18) with a positivity preserving piecewise linear reconstruction (2.15) for $\rho$. Assume that the system of ODEs (2.14) is discretized by the forward Euler (or a strong stability preserving Runge-Kutta) method. Then, the computed cell averages $\bar{\rho}_{j, k} \geq 0, \forall j, k$, provided the following CFL condition is satisfied:

$$
\Delta t \leq \min \left\{\frac{\Delta x}{4 a}, \frac{\Delta y}{4 b}\right\}, \quad a=\max _{j, k}\left\{u_{j+\frac{1}{2}, k}^{+},-u_{j+\frac{1}{2}, k}^{-}\right\}, \quad b=\max _{j, k}\left\{v_{j, k+\frac{1}{2}}^{+},-v_{j, k+\frac{1}{2}}^{-}\right\},
$$

where $u_{j+\frac{1}{2}, k}^{ \pm}$and $v_{j, k+\frac{1}{2}}^{ \pm}$are defined according to (2.6).

Discrete Entropy Dissipation. We define the discrete entropy

$$
E_{\Delta}(t)=\Delta x \Delta y \sum_{j, k}\left[\frac{1}{2} \Delta x \Delta y \sum_{i, l} W_{j-i, k-l} \bar{\rho}_{i, l} \bar{\rho}_{j, k}+H\left(\bar{\rho}_{j, k}\right)+\bar{V}_{j, k} \bar{\rho}_{j, k}\right],
$$

and discrete entropy dissipation

$$
I_{\Delta}(t)=\Delta x \Delta y \sum_{j, k}\left[\left(u_{j+\frac{1}{2}, k}\right)^{2}+\left(v_{j, k+\frac{1}{2}}\right)^{2}\right] \min _{j, k}\left(\rho_{j, k}^{\mathrm{E}}, \rho_{j+1, k}^{\mathrm{W}}, \rho_{j, k}^{\mathrm{N}}, \rho_{j, k+1}^{\mathrm{S}}\right) .
$$

Similarly to the 1-D case, we can show the following dissipative property of the scheme.

Theorem 2.8. Consider the system (1.1) with no flux boundary conditions in the domain $[-L, L]^{2}$ with $L>0$ and with initial data $\rho_{0}(x) \geq 0$. Given the semi-discrete finite-volume scheme (2.14), (2.17)-(2.18) with a positivity preserving piecewise linear reconstruction (2.15) for $\rho$, with $\Delta x=L / M$, and with discrete no-flux boundary conditions $F_{M+\frac{1}{2}, k}^{x}=F_{-M-\frac{1}{2}, k}^{x}=$ $F_{j, M+\frac{1}{2}}^{y}=F_{j,-M-\frac{1}{2}}^{y}=0$. Then,

$$
\frac{d}{d t} E_{\Delta}(t) \leq-I_{\Delta}(t), \quad \forall t>0
$$

\section{$3 \quad$ Numerical Experiments}

In this section, we present several numerical examples, focusing mainly on the steady states or long time behaviors of the solutions to the general equation

$$
\rho_{t}=\nabla \cdot\left[\rho \nabla\left(H^{\prime}(\rho)+V(\mathbf{x})+W * \rho\right)\right], \quad \mathbf{x} \in \mathbb{R}^{d}, t>0 .
$$

A previous detailed study in [5] for the degenerate parabolic and drift-diffusion equations demonstrated the good performance of the method (with small variants) in dealing with exponential rates of convergence toward compactly supported Barenblatt solutions. Here we will 
concentrate mostly on cases with the interaction potential $W$, and show that key properties like non-negativity and entropy dissipation are preserved. We will first start our discussion by using some test cases to validate the order of convergence of the scheme in space and its relation to the regularity of the steady states. If the solution $\rho$ is smooth, the spatial discretization given in Section 2 is shown to be of second order. However, in practice, the steady states of (1.1) are usually compactly supported, with discontinuities in the derivatives or even in the solutions themselves near the boundaries. This loss of regularity of the steady states usually leads to degeneracy in the order of convergence, as shown in Examples 1-4. Then, we will illustrate with several examples that the presented finite-volume scheme can be used for a numerical study of many challenging questions in which theoretical analysis has not yet been fully developed.

\subsection{Steady states: Spatial Order and Time Stabilization}

Example 1 (Attractive-repulsive kernels). We first consider equation (1.1) in 1-D with only the interaction kernel $W(x)=|x|^{2} / 2-\log |x|$ (i.e., $\left.H(\rho)=0, V(x)=0\right)$. The corresponding unit-mass steady state is given by (see [32]):

$$
\rho_{\infty}(x)= \begin{cases}\frac{1}{\pi} \sqrt{2-x^{2}}, & |x| \leq \sqrt{2} \\ 0, & \text { otherwise }\end{cases}
$$

and is Hölder continuous with exponent $\alpha=\frac{1}{2}$. This steady state is the unique global minimizer of the free energy (1.2) and it approached by the solutions of (1.1) with an exponential convergence rate as shown in [16]. We compute $\rho_{\infty}$ by numerically solving (1.1) at large time, with the initial condition $\rho(x, 0)=\frac{1}{\sqrt{2 \pi}} e^{-x^{2} / 2}$. In Figure 3.1(a), we plot the numerical steady state obtained on a very coarse grid with $\Delta x=\sqrt{2} / 5$. As one can see, even on such a coarse grid, the numerical steady state is in good agreement with the exact one, except near the boundary $x= \pm \sqrt{2}$. The spatial convergence error of the steady states in $L^{1}$ norm and $L^{\infty}$ norm is shown in Figure 3.1(b). As a general rule, the practical convergence error of the numerical steady state is $\alpha$ in $L^{\infty}$ norm and $\alpha+1$ in $L^{1}$ norm, if the exact steady state is $C^{\alpha}$-Hölder continuous.

Example 2 (Nonlinear diffusion with nonlocal attraction kernel). Next, we consider the equation (1.1) in 1-D with $H(\rho)=\frac{\nu}{m} \rho^{m}, W(x)=W(|x|)$ and $V(x)=0$, where $\nu>0, m>1$ and $W \in \mathcal{W}^{1,1}(\mathbb{R})$ is an increasing function on $[0, \infty)$, i.e.,

$$
\rho_{t}=\left(\rho\left(\nu \rho^{m-1}+W * \rho\right)_{x}\right)_{x} .
$$

This equation arises in some physical and biological modelling with competing nonlinear diffusion and nonlocal attraction, see [35] for instance. The attraction represented by convolution $W * \rho$ is relatively weak (compared to that in the Keller-Segel model discussed below), and the solution does not blow up with bounded initial data, while the long time behavior of the solution is characterized by an extensive study of the steady states in [11]. When $m>2$, the attraction dominates the nonlinear diffusion, leading to a compactly supported steady state. When $m<2$, the behavior depends on the diffusion coefficient $\nu$ : there is a local steady state for small $\nu$ with localized initial data and the solution always decays to zero for large $\nu$. The borderline case $m=2$ is investigated in [10] for non-compactly supported kernels, where the evolution depends on the coefficient $\nu$, the total conserved mass, and $\|W\|_{1}$. 

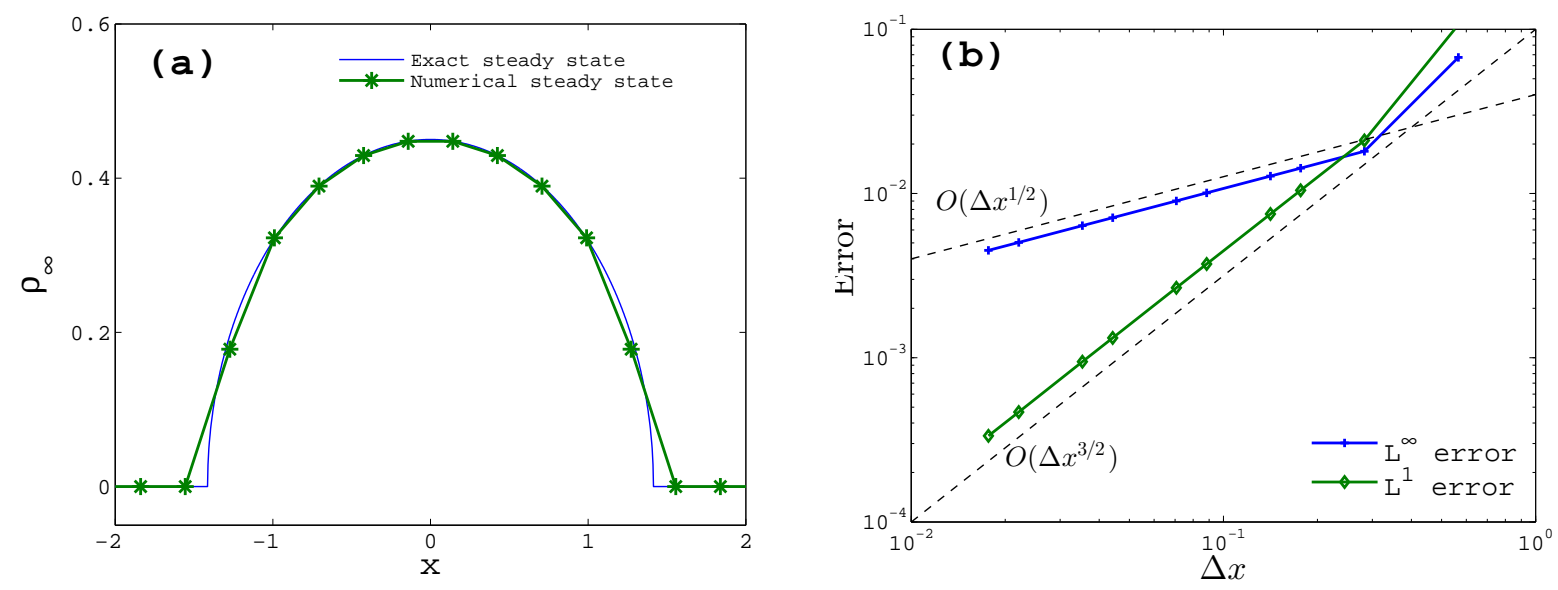

Figure 3.1: (a) The numerical steady state with grid size $\Delta x=\sqrt{2} / 5$, compared with the exact one. (b) The convergence of error in $L^{1}$ and $L^{\infty}$ norms. Here the $L^{1}$ norm is computed by taking the numerical steady state piecewise constant inside each cell and $L^{\infty}$ norm is evaluated only at the cell centers.

We begin by numerically calculating the solutions to the 1-D equation (3.1) with nonlinear diffusion and $W(x)=-e^{-|x|^{2} / 2 \sigma} / \sqrt{2 \pi \sigma}$, for some constants $\sigma>0$. The corresponding steady states can also be obtained by implementing an iterative procedure proposed in [11]. Here, we compute the steady state solutions $\rho_{\infty}$ by the time evolution of (3.1) subject to Gaussian-type initial data

$$
\rho_{0}(x)=\frac{1}{\sqrt{8 \pi}}\left[e^{-0.5(x-3)^{2}}+e^{-0.5(x+3)^{2}}\right] .
$$

The simulations are run on the computational domain $[-6,6]$ with the mesh size $\Delta x=0.02$ for large time until stabilization and the results are plotted in Figure 3.2(a) for different values of $m$. As one can observe, the boundary behavior of the compactly supported steady states has a similar dependence on $m$ as the Barenblatt solutions of the classical porous medium equation $\rho_{t}=\nu\left(\rho\left(\rho^{m-1}\right)_{x}\right)_{x}$, that is, only Hölder continuous with exponent $\alpha=\min (1,1 /(m-1))$. Using the steady states of (1.1) computed by the iterative scheme proposed in [11], we can check the spatial convergence error of our scheme on different grid sizes $\Delta x$. As shown in Figure 3.2(b), the spatial convergence error of the steady states is $\min (2, m /(m-1))$ in $L^{1}$ norm and is $\min (1,1 /(m-1))$ in $L^{\infty}$ norm.

Now let us turn our attention to the time evolution and the stabilization in time toward equilibria and show that the convergence in time toward equilibration can be arbitrarily slow. This is due to the fact that the effect of attraction is very small for large distances. Actually, different bumps at large distances will slowly diffuse and take very long time to attract each other. However, once they reach certain distance, the convexity of the Gaussian well will lead to equilibration exponentially fast in time. These two different time scales can be observed in Figure 3.3, where the time energy decay and density evolution are plotted to the solution corresponding to $m=3, \sigma=1$, and $\nu=1.48$ (see also Figure 3.2).

Example 3 (Nonlinear diffusion with compactly supported attraction kernel). The dynamics of the solution of the 1-D equation (1.1) with characteristic functions as initial data is shown in Figure 3.4, for the compactly supported interaction kernel $W(x)=-(1-|x|)_{+}$. 

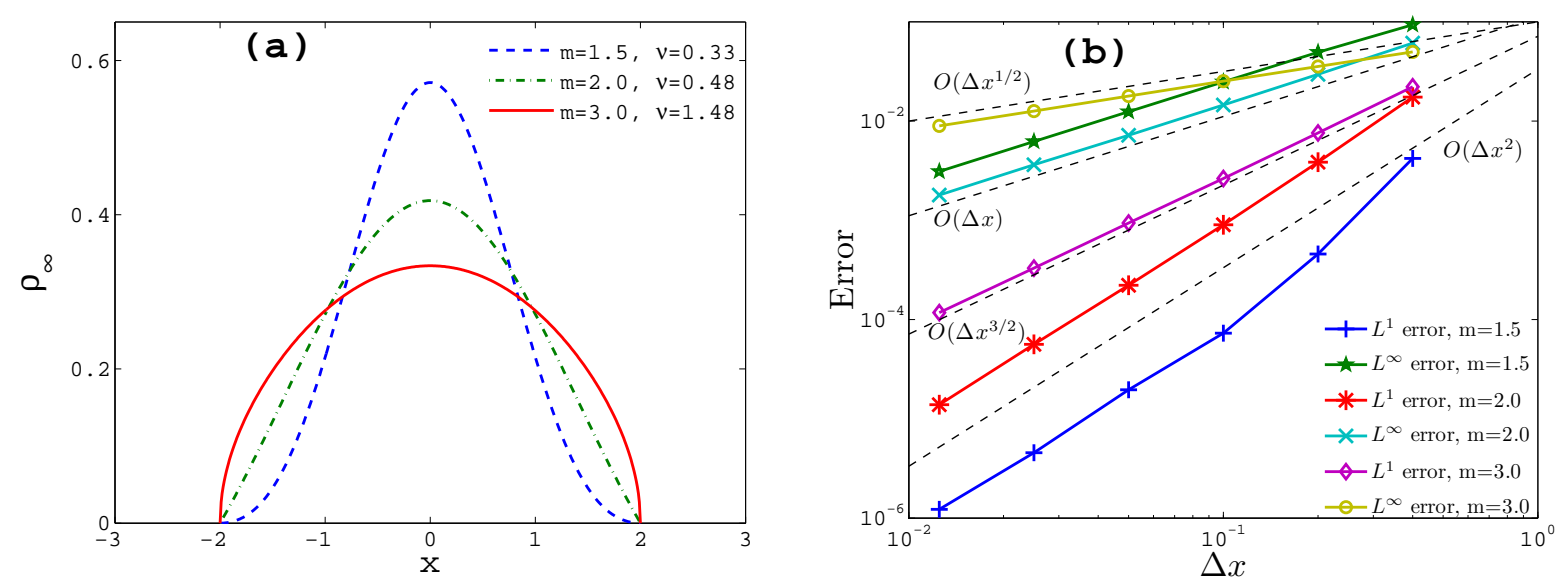

Figure 3.2: (a) The steady states with unit total mass for different $m$ have Hölder exponent $\alpha=\min (1,1 /(m-1))$ and $\sigma=1$, where $\nu$ is chosen such that the corresponding $\rho_{\infty}$ is supported on $[-2,2]$. (b) The convergence of the steady states $\rho_{\infty}$ on different grid size $\Delta x$, which is $\min (2, m /(m-1))$ in $L^{1}$ norm and is $\min (1,1 /(m-1))$ in $L^{\infty}$ norm.

For $\rho_{0}(x)=\chi_{[-2,2]}(x)$, the solution forms two bumps and then merges to a single one, which is the global minimizer of the energy. When $\rho_{0}(x)=\chi_{[-3,3]}(x)$, the solution converges to three non-interacting bumps (in the sense that $\frac{\partial \xi}{\partial x} \rho \equiv 0$ ), each of which is a steady state.

The decay of the energy for these two cases is shown in Figure 3.5(a). After the initial transient disappears, the energy decreases significantly at later times only when the topology changes, i.e. the merge of disconnected components. Although there is a steady state with one single component with all the mass, the three-bump solutions with $\rho_{0}(x)=\chi_{[-3,3]}$ seems to be the correct final stable steady state. This can be confirmed from Figure 3.5(b), as $\xi$ is a constant on each connected component of the support.

This example shows a very interesting effect in this equation, which is the appearance in the long time asymptotics of steady states with disconnected support. It should be observed that each bump is at distance larger than 1 from the other bumps, and thus the interaction force exerted between them is zero. This together with the finite speed of propagation of the degenerate diffusion are the reasons why the steady state with the total mass and connected support is not achieved in the long time asymptotics. This fact is related to the existence of local minimizers of the functional (1.2) in certain weak topologies, infinity Wasserstein distance, not allowing for large perturbations of the support, see [2, Section 5] and [22] for related questions.

For other non-compactly supported kernels like $W(x)=-\frac{1}{2} e^{-|x|}$ or the Gaussian as in Example 2, there is a unique steady state with one single connected component in its support, though it exhibits the same slow-fast behavior in its convergence in time as shown in Figure 3.3. This metastability and other decaying solutions when $m<2$ are discussed in more details in $[11]$.

Example 4 (Nonlinear diffusion with double well external potential). In this example, we elaborate more on stationary states which are not global minimizers of the total energy. More precisely, we consider nonlinear diffusion equation for particles under an external double-well 

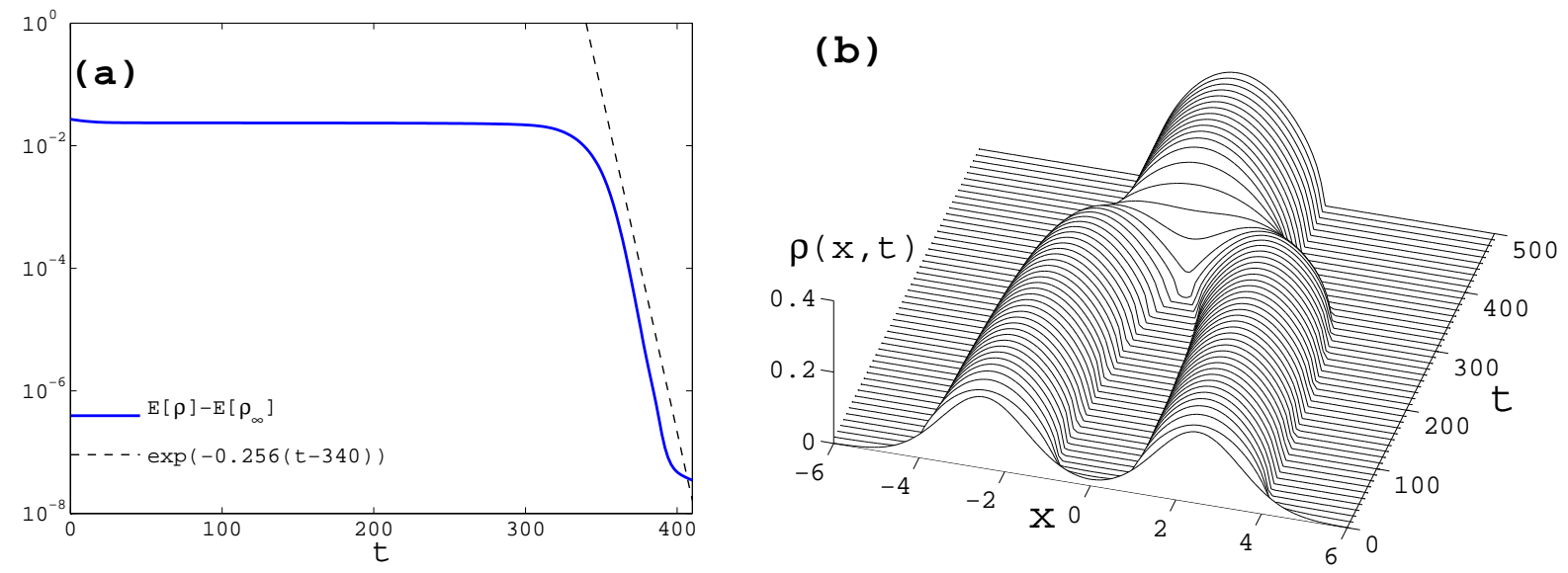

Figure 3.3: (a) The two timescales in the decay towards the unique equilibrium solution corresponding: very slow energy decay followed by an exponential decay. (b) Time evolution of the density. Here, $m=3, \sigma=1$ and $\nu=1.48$.
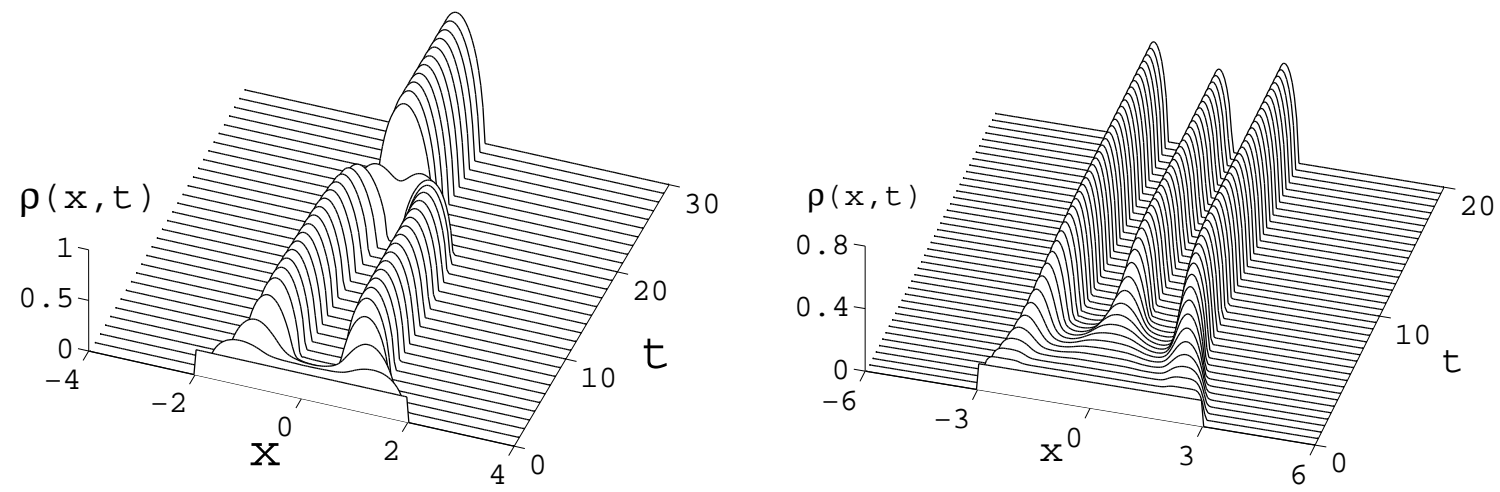

Figure 3.4: The dynamics of (3.1) starting with the initial data $\rho_{0}=\chi_{[-2,2]}$ and $\rho_{0}=\chi_{[-3,3]}$.

potential of the form

$$
\rho_{t}=\left(\rho\left(\nu \rho^{m-1}+V\right)_{x}\right)_{x}, \quad V(x)=\frac{x^{4}}{4}-\frac{x^{2}}{2} .
$$

Actually, the steady states of (3.2) are of the form

$$
\rho_{\infty}(x)=\left(\frac{C(x)-V(x)}{\nu}\right)_{+}^{\frac{1}{m-1}}
$$

with $C(x)$ piecewise constant possibly different in each connected component of the support.

We run the computation with $\nu=1, m=2$ and initial data of the form

$$
\rho_{0}(x)=\frac{M}{\sqrt{2 \pi \sigma^{2}}} e^{-\frac{\left(x-x_{c}\right)^{2}}{2 \sigma^{2}}}, \quad M=0.1, \sigma^{2}=0.2,
$$

corresponding to the symmetric $\left(x_{c}=0\right)$ and asymmetric $\left(x_{c}=0.2\right)$ cases, respectively. It is obvious that for small mass, we can get infinitely many stationary states with two connected 

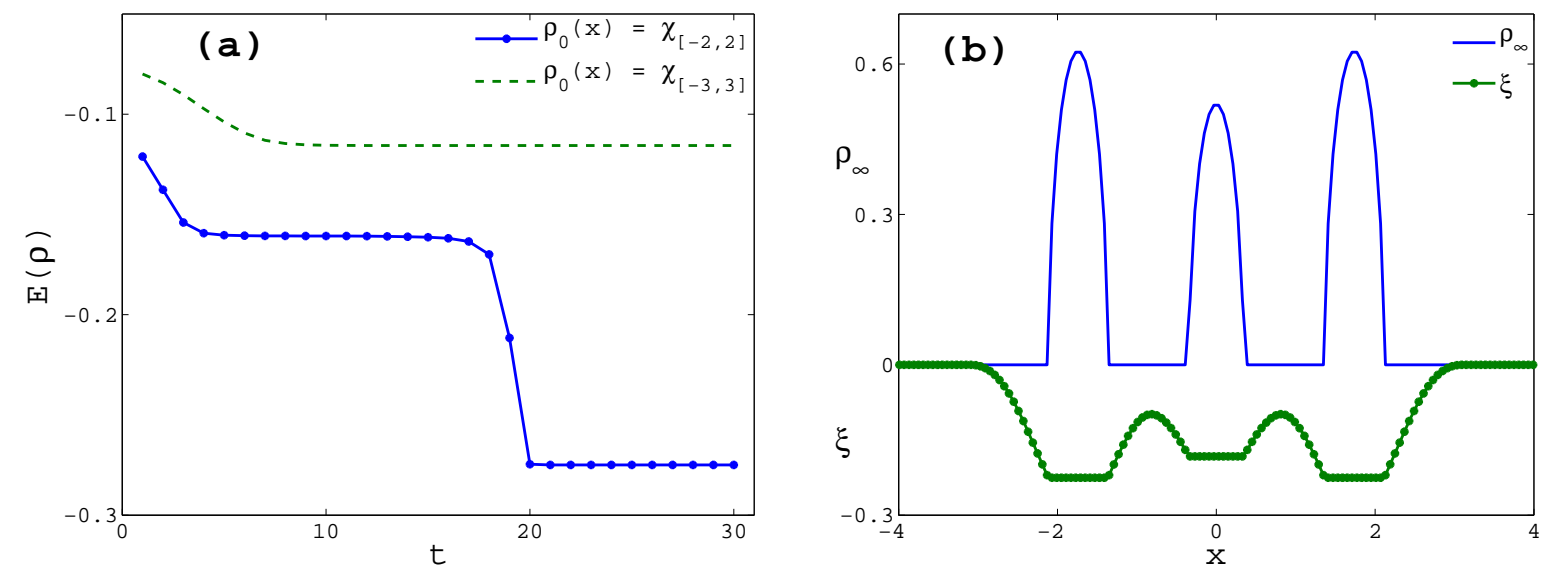

Figure 3.5: (a) The decay of the entropy of the equation $(3.1)$ with initial data $\rho_{0}(x)=\chi_{[-2,2]}$ and $\rho_{0}(x)=\chi_{[-3,3]}$. After an initial transient behavior, there is a significant decrease in the entropy only when the topology of the solution changes. (b) The final steady state of (3.1) with initial data $\rho_{0}(x)=\chi_{[-3,3]}$ and the corresponding $\xi$. Here $\xi$ assumes different constant values on different connected components of the support.

components in its support by perturbing the value of $C$ defining a symmetric steady state. Actually, each of them has a non zero basin of attraction depending on the distribution of mass initially as shown in Figure 3.6(b). While the global minimizer of the free energy is the symmetric steady state, the non symmetric ones are local minimizers in the infinity Wassertein distance or informally for small perturbations in the sense of its support. It is interesting to observe that even if the long time asymptotics is different for each initial data, the rate of convergence to stabilization seems uniformly 2, see Figure 3.6(a).

\subsection{Generalized Keller-Segel model}

Another related diffusion equation with nonlocal attraction is the generalized Keller-Segel model,

$$
\rho_{t}=\nabla \cdot\left(\rho \nabla\left(\nu \rho^{m-1}+W * \rho\right)\right),
$$

with the kernel $W(\mathbf{x})=|\mathbf{x}|^{\alpha} / \alpha$ with $-d<\alpha$ or the convention $W(\mathbf{x})=\ln |\mathbf{x}|$ for $\alpha=0$. The bound from below in $\alpha$ due to local integrability of the kernel $W$. When $\alpha=2-d, W$ is the Newtonian potential in $\mathbb{R}^{d}$, and the equation reduces to the Keller-Segel model for chemotaxis with nonlinear diffusion:

$$
\rho_{t}=\nabla \cdot\left(\rho \nabla\left(\nu \rho^{m-1}-c\right)\right), \quad-\Delta c=\rho .
$$

Compared with Example 2 where the interaction potential $W$ is integrable, the long tail for $W(\mathbf{x})=|\mathbf{x}|^{\alpha} / \alpha$ has non-trivial consequences. In certain parameter regimes, the solution can even blow up in finite time with smooth initial data. To clarify the different regimes, we can easily evaluate the balance between the attraction due to the nonlocal kernel $W$ and the repulsion due to diffusion by scaling arguments. In fact, taking the corresponding energy functional (1.2) and checking the scaling under dilations of each term, we can find three different regimes: 

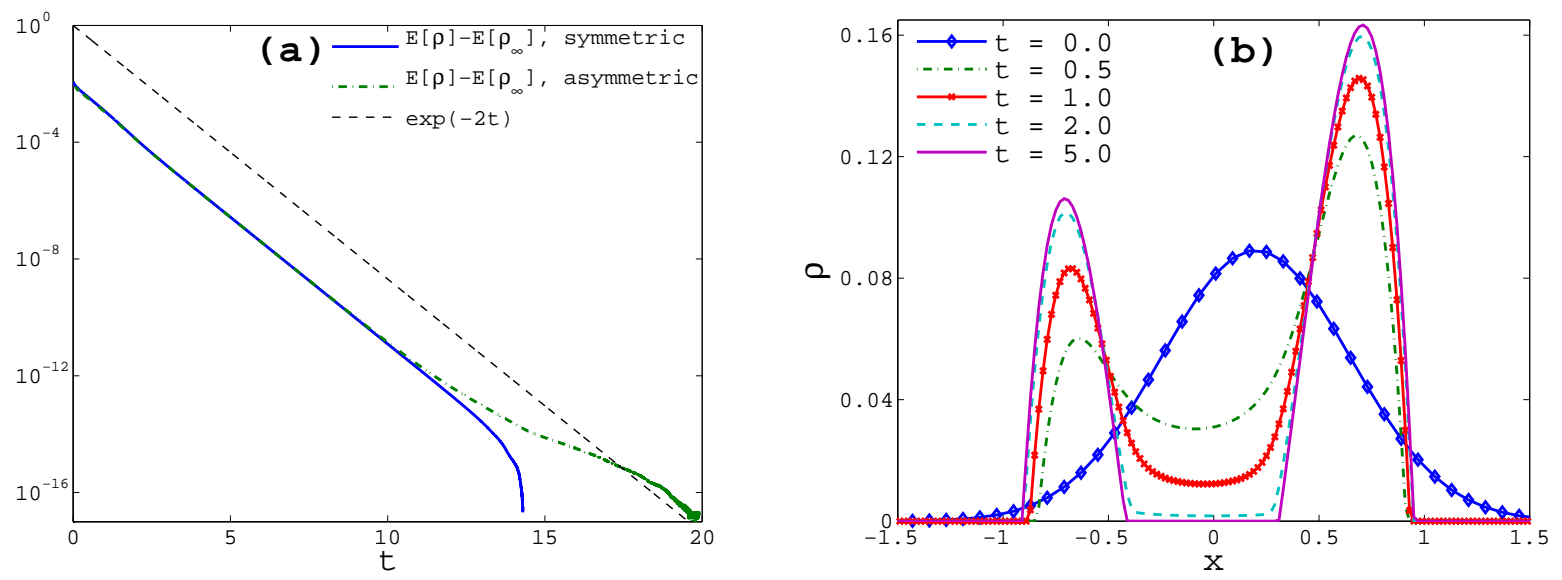

Figure 3.6: (a) The decay of the entropy of the equation (3.2) with initial data (3.3), for the symmetric $\left(x_{c}=0\right)$ and asymmetric $\left(x_{c}=0.2\right)$ cases, respectively. A uniform rate of convergence of order 2 is observed towards the stationary states. (b) The evolution of the asymmetric initial data $\left(x_{c}=0.2\right)$ towards the corresponding asymmetric stationary state.

- Diffusion-dominated regime: $m>(d-\alpha) / d$. Here, the intuition is that solutions exist globally in time and the aggregation effect only shows in the long-time behavior where we observe nontrivial compactly supported stationary states.

- Balanced regime: $m=(d-\alpha) / d$. Here the mass of the system is the critical quantity. There is a critical mass, separating the diffusive behavior from the blow-up behavior.

- Aggregation-dominated regime: $m<(d-\alpha) / d$. Blow-up and diffusive behavior coexist for all values of the mass depending on the initial concentration.

The classical 2-D Keller-Segel system corresponds to $m=1, \alpha=0$, see $[6,8,12,39]$ and the references therein for the different behaviors. The nonlinear diffusion model for the balanced case with the Newtonian potential in $d \geq 3$ was studied in detail in [7]. Finite time blow-up solutions for general kernel $W(\mathbf{x})=|\mathbf{x}|^{\alpha} / \alpha$ in the aggregation-dominated regime were also investigated, combined with numerical simulations [42].

Example 5 (Generalized Keller-Segel model in the balanced regime). Let us start with the 1-D example when $m+\alpha=1$ corresponding to the balanced case. Here, the behavior of the dynamics depends on the total conserved mass. The solutions blow up if the mass is greater than the threshold $M_{c}$ and otherwise the solutions decay to zero. This threshold mass can be determined by solving the equation with different initial conditions and is shown in Figure 3.7(a) for different values of $m$. For example, when $m=1.5$ and $\alpha=1-m=-0.5$, the threshold mass $M_{c}$ is about 0.055. If the initial data has a larger mass as in Figure 3.7(b), the solution blows up. Since the numerical method is conservative, the density concentrates inside one cell instead of being infinity. Otherwise, if the initial data has a smaller mass as in Figure 3.7(c), the solution decays to zero.

We have also checked the self-similar behavior for subcritical mass cases $\left(M<M_{c}\right)$ in the sense of solving (3.4) with $V(x)=|x|^{2} / 2$. That is in the similarity variables, the solution of $\rho_{t}=\nabla \cdot\left(\rho \nabla\left(\nu \rho^{m-1}+W * \rho+|x|^{2} / 2\right)\right)$ converges to the self-similar profile. The decay rate in time is computed for several subcritical masses and is shown in Figure 3.8(a), illustrating 

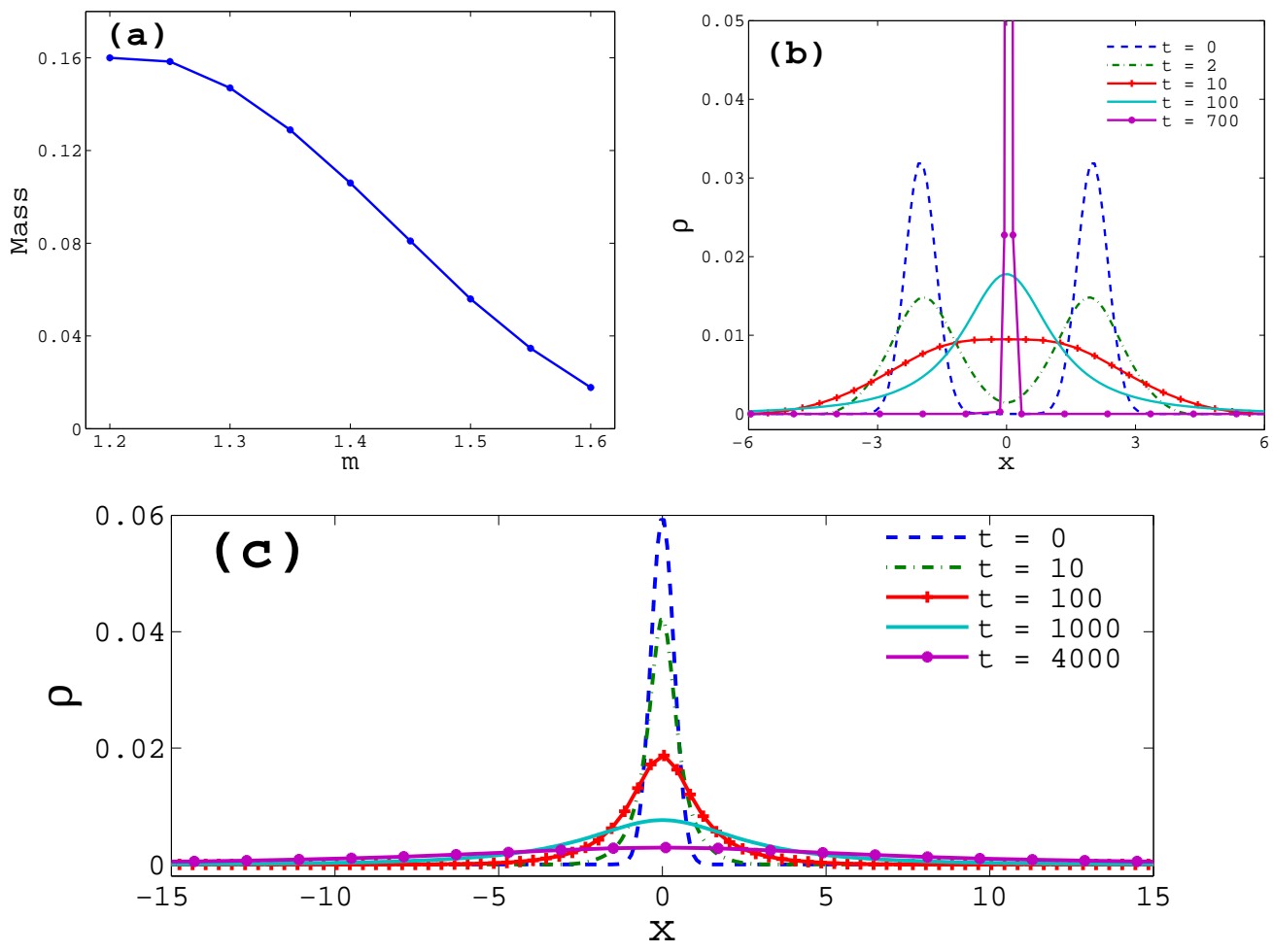

Figure 3.7: (a) The critical mass $M_{c}$ when $m+\alpha=1, \nu=1$ for different exponents $m$. (b) The blowing up solution for $m=1.5, \alpha=-0.5$ and $\nu=1$ with initial data $\rho_{0}(x)=$ $M\left(e^{-4(x+2)^{2}}+e^{-4(x-2)^{2}}\right) / \sqrt{\pi}$, where the total mass $M=0.057>M_{c} \approx 0.055$. (c) The decaying solution for $m=1.5, \alpha=-0.5$ and $\nu=1$ with initial data $\rho_{0}(x)=M e^{-x^{2}} / \sqrt{\pi}$, where $M=0.53<M_{c}=0.55$.

that this rate is independent of the mass and is exactly $O\left(e^{-2 t}\right)$ as proven in the classical 2-D Keller-Segel model in [13]. We also observe in Figure 3.8(b) how the self-similar profiles become concentrated as a Dirac Delta at the origin as $M \rightarrow M_{c}$.

Example 6 (Generalized Keller-Segel model in the other regimes). The general behaviors of solutions to the 1-D version of (3.4) in other parameter regimes are also known to some extent. When $m>1-\alpha$ corresponding to the diffusion-dominated regime, a compact steady state is always expected, which is the global minimizer of the energy (1.2) as in [33]. If the nonlinearity of the diffusion is increased to be $m=1.6$ with the same total mass $(=0.057)$ and the exponent $\alpha=-0.5$, the solution converges to a steady state as in Figure 3.9 instead of blowing up as in Figure 3.7(b). When $\alpha+m<1$ corresponding to the aggregation-dominated regime, the small initial data decays to zero while large initial data blows up in finite time (see Figure 3.10). The size of the initial data determining the distinct behaviors is usually measured in a norm different from $L^{1}$ (the conserved mass), and no critical value in this norm as in the case $m+\alpha=1$ is expected. 

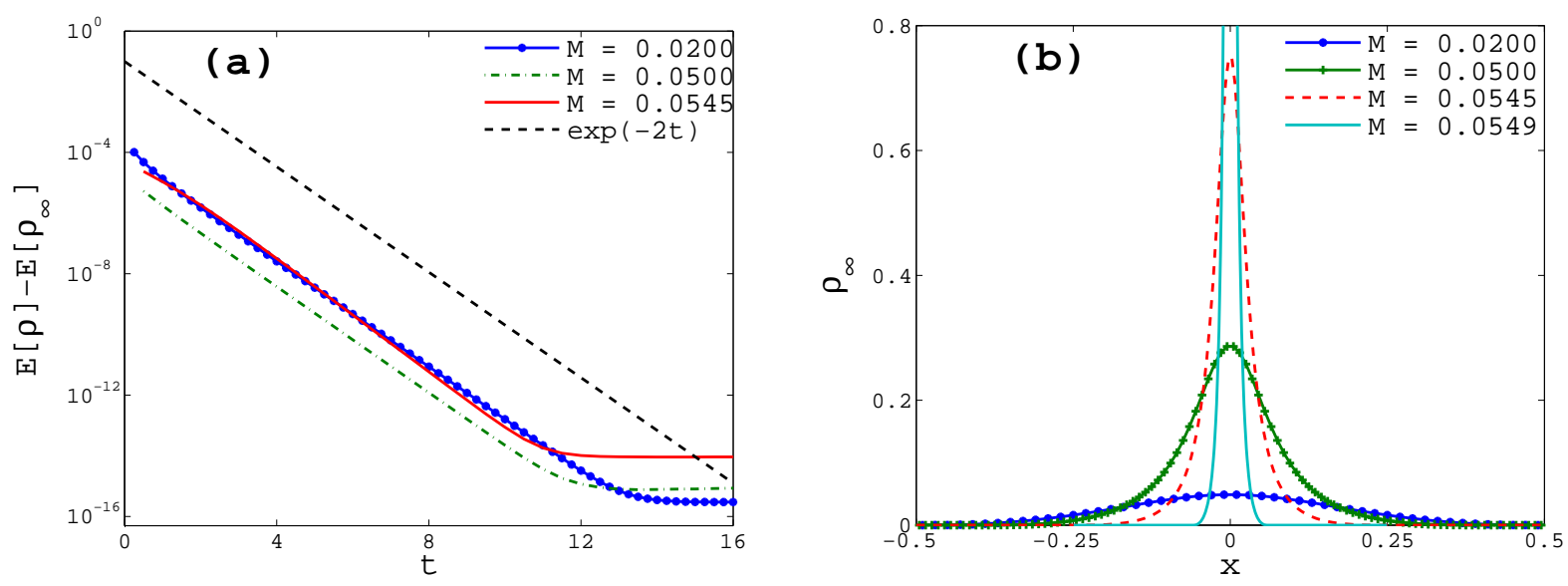

Figure 3.8: (a) The uniformly exponential decay towards equilibrium (in similarity variables) for subcritical mass in self-similar variables when $m=1.5, \alpha=-0.5, \nu=1$ for different values of the mass $M<M_{c}$. (b) The equilibrium profiles for different $M<M_{c}$.
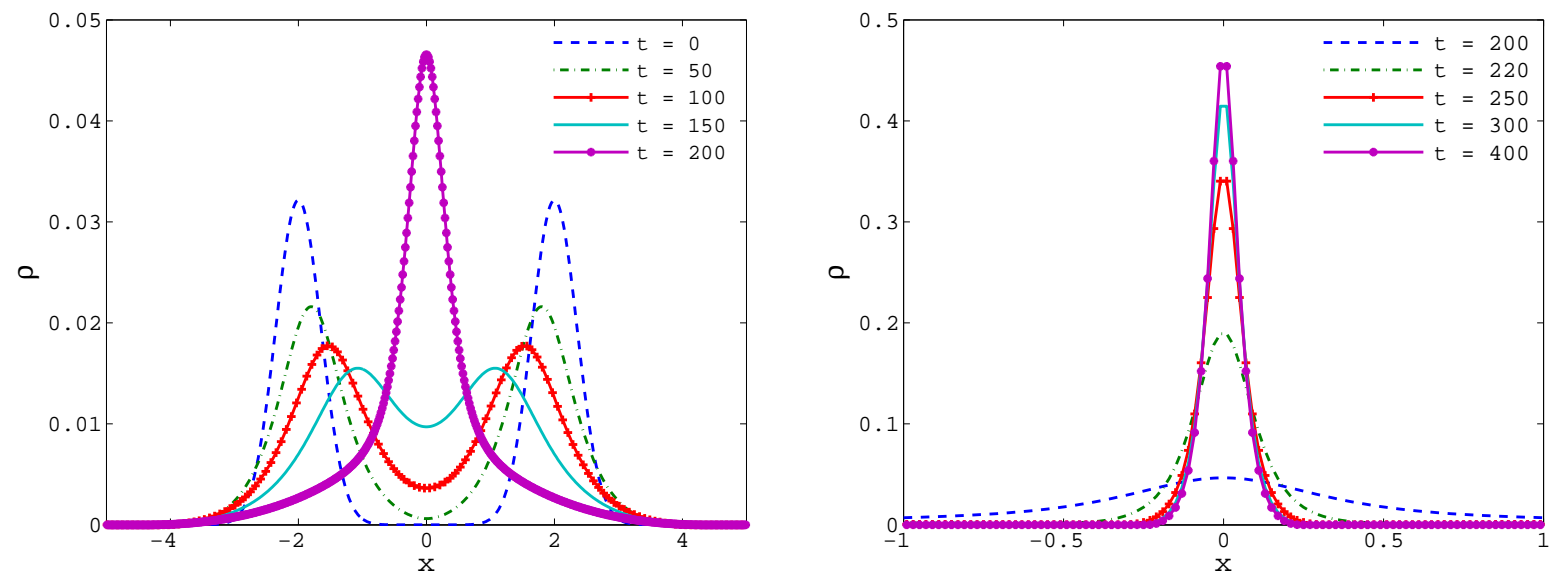

Figure 3.9: The evolution of the generalized Keller-Segel equation in the diffusion dominated regime $(m=1.6, \alpha=-0.5)$ with $\nu=1.0$. The initial condition $\rho_{0}(x)=M\left(e^{-4(x+2)^{2}}+\right.$ $\left.e^{-4(x-2)^{2}}\right) / \sqrt{\pi}(M=0.057)$ is the same as that in Figure $3.7(\mathbf{b})$. 

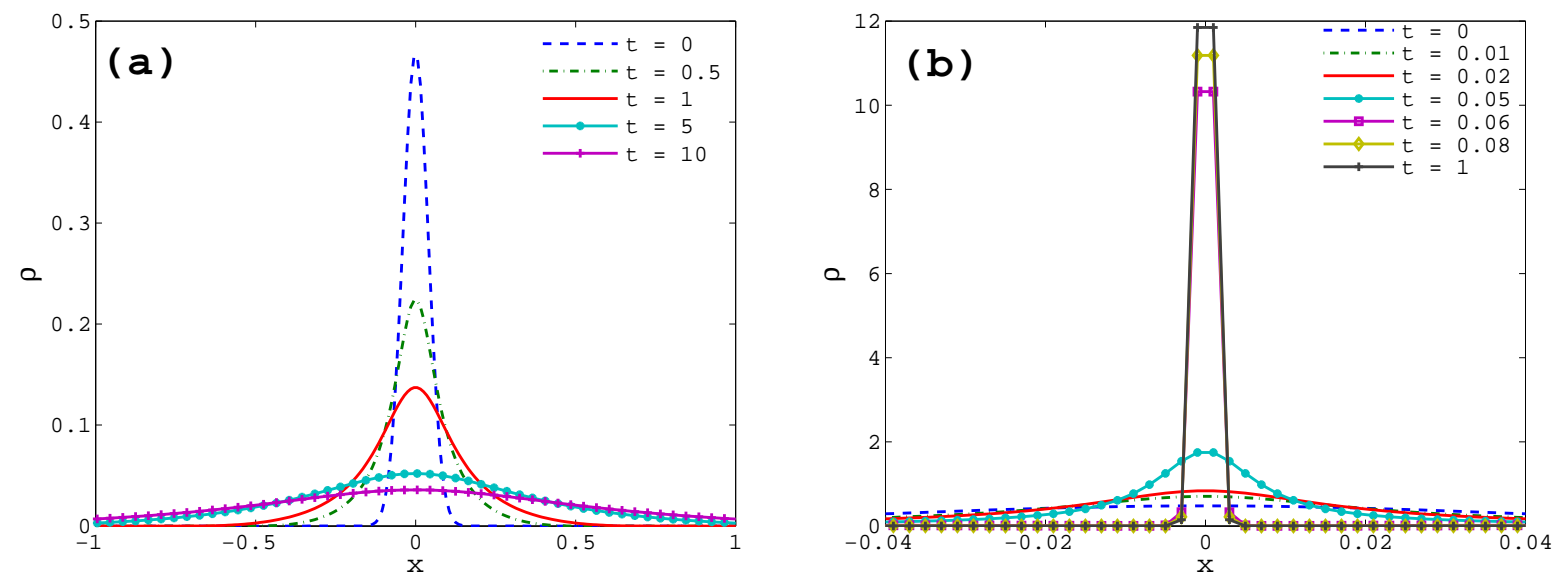

Figure 3.10: The evolution of the generalized Keller-Segel equation in the aggregationdominated regime $(m=1.6, \alpha=-0.5)$ with $\nu=1.0$. The initial condition is $\rho_{0}(x)=$ $M\left(e^{-4(x+2)^{2}}+e^{-4(x-2)^{2}}\right) / \sqrt{\pi}$, with $M=0.047$ for decaying solution in (a) and $M=0.048$ for blowup solutions in (b).

\subsection{Aggregation equation with repulsive-attractive kernels}

In the absence of diffusion from $H(\rho)$ or confinement from $V$, steady states of the general equation (1.1) are still expected when the kernel $W$ incorporates both short range repulsion and long range attraction. This type of kernels arises in the continuum formulation of moving flocks of self-propelled particles [20,26], and the popular ones are the Morse potential

$$
W(x)=C e^{-|x| / \ell}-e^{-|x|}, \quad C>1, \ell<1
$$

and the power-law type

$$
W(x)=\frac{|x|^{a}}{a}-\frac{|x|^{b}}{b}, \quad a>b,
$$

with the convention that $|x|^{0} / 0=\ln |x|$ below.

Example 7 (Quadratic attractive and Newtonian repulsive kernels). The regularity of the solution depends on the singularity of the repulsion force. If this force is small at short distance (or equivalently $b$ is relatively large), the solution can concentrate at a lower dimension subset, while more singular forces lead to smooth steady states except possible discontinuities near the boundary [2]. The case $a=2$ and $b=0$ is shown in Example 1, whose steady state is a semi-circle [16,32], while the case $a=2$ and $b=1$ leads to a steady state which is a constant supported on an interval [21,23].

We remind that the discrete convolution for the velocity field in (2.8) is discretized using the coefficients $W_{j-i}$, chosen as approximations of the local integral

$$
W_{j-i}=\frac{1}{\Delta x} \int_{C_{i}} W\left(x_{j}-s\right) d s .
$$

In the case of smooth kernels $(b>0)$, we can either use the mid-point rule or a direct computation of the integral if available. We show the numerically computed stationary state with both 
options in Figure 3.11 (a) and (b) respectively. As one can observe, the first choice is oscillation free while the second choice with exact integrals shows an overshoot of the density near the boundary of the support. The difference between the two cases can be explained by carefully writing down the characterization of the discrete stationary states based on the discrete entropy inequality in Theorem 2.5. The mid-point rule performs better due to its symmetry that induces some numerical diffusion.
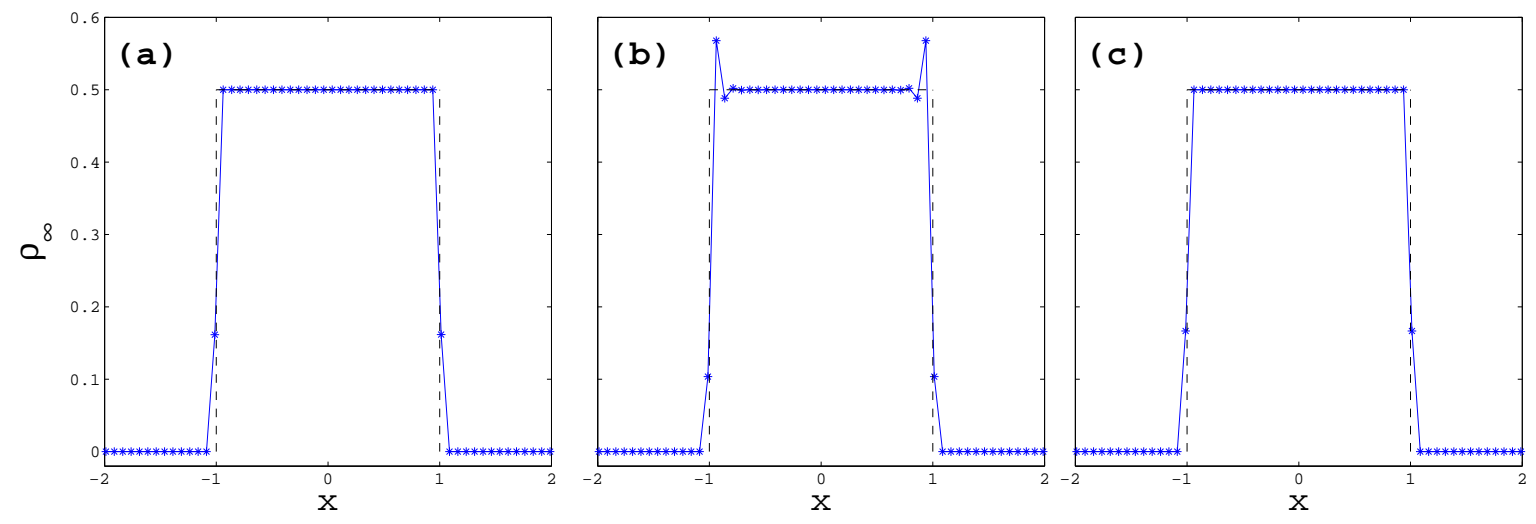

Figure 3.11: The steady states computed with: (a) mid-point quadrature rule for (3.6); (b) exact computation of $W_{j-i}$ in (3.6); (c) Same as (b) but adding small nonlinear diffusion.

In case we would be dealing with singular kernels, we cannot use simple quadrature formulas like middle-point rule but rather we need to implement either quadrature formulas for singular integrals or perform exact evaluations of the integrals in (3.6). To avoid the oscillations as in Figure 3.11(b), we added a small nonlinear diffusion term, i.e., $\rho_{t}=\left(\rho(\epsilon \rho+W * \rho)_{x}\right)_{x}$. Here quadratic nonlinear diffusion is used, respecting the same nonlinearity and scaling as in the original equation $\rho_{t}=\left(\rho(W * \rho)_{x}\right)_{x}$. Numerical experiments as in Figure 3.11(c) indicate that $\epsilon=0.25(\Delta x)^{2}$ is close to optimal, in the sense that $\epsilon$ is just large enough to prevent the overshoot. This near optimal diffusion coefficient has been further confirmed by numerical experiments with different $\Delta x$.

For the sake of clarity, we show in Figure 3.12(a)-(b) the steady-state solutions computed on a finer mesh for the same cases as in Figure 3.11(a)-(b) along with the $O(\Delta x)$ decay of $L^{1}$ errors for different grid sizes $\Delta x$ in Figure 3.12(c). The $L^{\infty}$ errors is almost constant and not decaying with mesh refinement. They clearly indicate that the overshoot amplitude seen in Figures 3.11(b) and 3.12(b) is not reduced by mesh refinement and it needs the fix of small diffusion regularization. This will be further discussed in 2-D simulations below.

\subsection{Two-dimensional simulations}

Now, let us illustrate the performance of the scheme in 2-D with some selected examples showcasing different numerical difficulties and interesting asymptotics.

Example 8 (Nonlinear diffusion with nonlocal attraction in 2-D). For the equation with $H(\rho)=\frac{\nu}{m} \rho^{m}, W(\mathbf{x})=-\exp \left(-|\mathbf{x}|^{2}\right) / \pi$ and $V \equiv 0$, the dynamics is similar to that in $1-\mathrm{D}$, being 

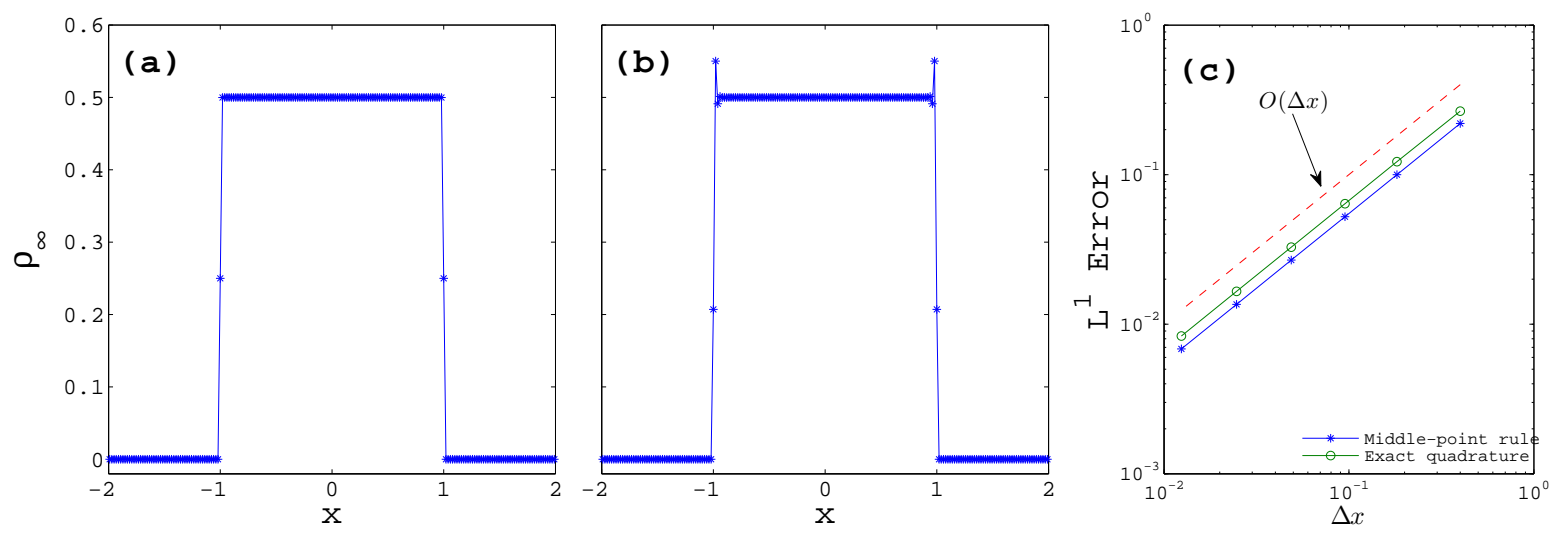

Figure 3.12: The steady states computed with on a finer mesh with: (a) mid-point rule for (3.6); (b) exact computation of $W_{j-i}$; (c) the convergence of $L^{1}$ errors for both options.
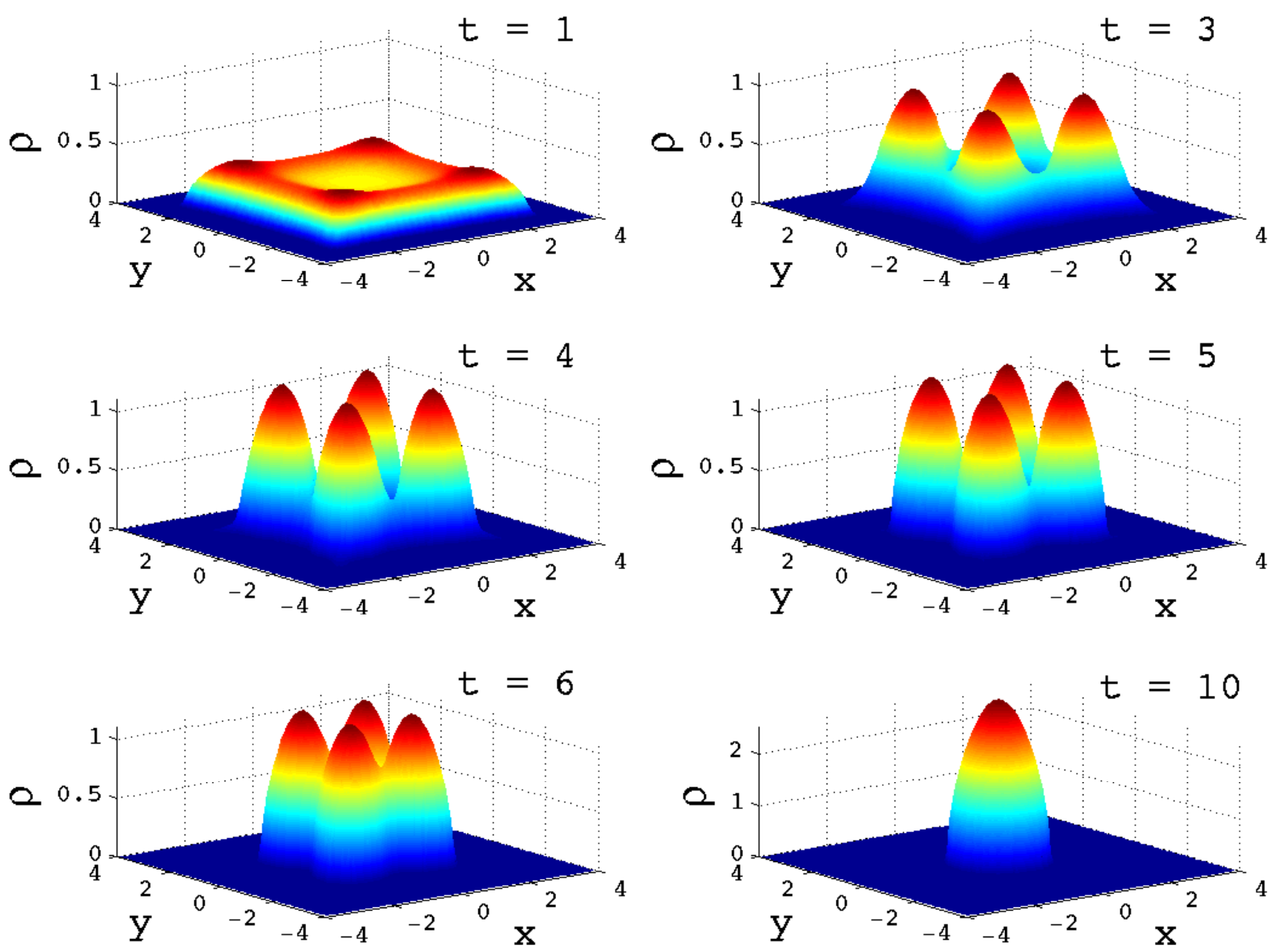

Figure 3.13: The evolution of the 2 d aggregation equation with nonlinear diffusion with $\nu=0.1$, $m=3, W(\mathbf{x})=\exp \left(-|\mathbf{x}|^{2}\right) / \pi$ and initial condition $\rho_{0}(\mathbf{x})=\frac{1}{4} \chi_{[-3,3] \times[-3,3]}(\mathbf{x})$. The computational domain is $[-4,4] \times[-4,4]$, with grid size $\Delta x=\Delta y=0.1$ and time step $\Delta t=0.001$. 
the result of the competition between the nonlinear diffusion $\nabla \cdot\left(\rho \nabla\left(\nu \rho^{m-1}\right)\right)$ and the nonlocal attraction $\nabla \cdot(\rho \nabla W * \rho))$. The evolution starting from the rescaled characteristic function supported on the square $[-3,3] \times[-3,3]$ is shown in Figure 3.13. Because the interaction represented by the kernel $W(\mathbf{x})$ is nonzero for any $\mathbf{x}=(x, y)$, the final steady state consists of one single component; however, four clumps are formed in the evolution, as the attraction dominates the relatively weak diffusion.

Example 9 (Quadratic attractive and Newtonian repulsive kernel with small nonlinear diffusion). Similarly, overshoots may appear near the boundary of discontinuous solutions of $\rho_{t}=\nabla \cdot(\rho \nabla W * \rho)$ with repulsive-attractive kernels $W$. These overshoots can not be eliminated as easily as in one dimension, either by a careful choice of grid to align with the boundary or by a special numerical quadrature for $W_{i-j}$. However, stable solutions can be obtained by adding small nonlinear diffusion as in Example 7. Therefore, we consider the equation

$$
\rho_{t}=\nabla \cdot(\rho \nabla(\epsilon \rho+W * \rho)) .
$$

For quadratic attractive and Newtonian repulsive kernel $W(\mathbf{x})=|\mathbf{x}|^{2} / 2-\ln |\mathbf{x}|$, the steady states are shown in Figure 3.14, without $(\epsilon=0)$ or with the diffusion. The near optimal coefficient $\epsilon$ is numerically shown to be close to $0.4\left((\Delta x)^{2}+(\Delta y)^{2}\right)$, exhibiting a similar mesh dependence as in Example 7. Since $W$ is singular in this (and next) example, $W_{j, k}$ is computed using Gaussian quadrature with four points in each dimension, to avoid the evaluation of $W$ at the origin.

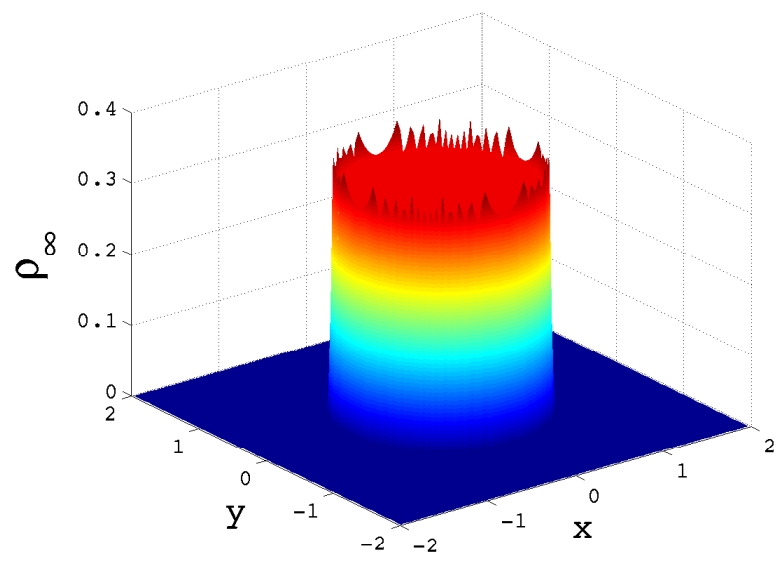

(a) $\epsilon=0$

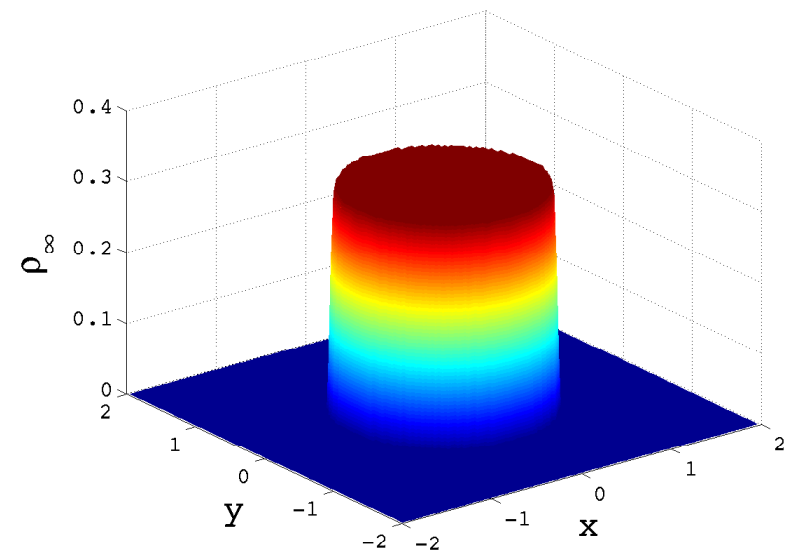

(b) $\epsilon=0.4\left((\Delta x)^{2}+(\Delta y)^{2}\right)$

Figure 3.14: (a) the steady state of the equation with $W(\mathbf{x})=|\mathbf{x}|^{2} / 2-\ln |\mathbf{x}|$; (b) the steady state with the same $W(\mathbf{x})$, regularized by quadratic diffusion $\nabla \cdot(\rho \nabla(\epsilon \rho))$. The exact steady state without diffusion is the characteristic function of the unit disk with density $\frac{1}{\pi}$.

Example 10 (Steady mill solutions). Another common pattern observed for the selfpropelled particle systems with an attractive-repulsive kernel in 2-D is the rotating mill [15], and the steady pattern can be obtained from the equation

$$
\rho_{t}=\nabla \cdot\left(\rho \nabla\left(W * \rho-\frac{\alpha}{\beta} \log |\mathbf{x}|\right)\right), \quad \mathbf{x} \in \mathbb{R}^{2},
$$


with some positive constants $\alpha$ and $\beta$. For the kernel $W(\mathbf{x})=\frac{1}{2}|\mathbf{x}|^{2}-\ln |\mathbf{x}|$, the steady state is still a constant $\rho_{\infty}=2$ on an annulus, whose inner and outer radius are given by

$$
R_{0}=\sqrt{\frac{\alpha}{\beta}}, \quad R_{1}=\sqrt{\frac{\alpha}{\beta}+\frac{M}{2 \pi}},
$$

with the total conserved mass $M=\int_{\mathbb{R}^{d}} \rho d \mathbf{x}$. For other more realistic kernels like the Morse type [15] or Quasi-Morse type [14], the radial density is in general more concentrated near the inner radius, but the explicit form of $\rho_{\infty}$ can not be obtained in general. Numerical diffusion, in the form of $\epsilon \nabla \cdot(\rho \nabla \rho)$, is still needed to prevent the overshoot and the resulting steady states with $\epsilon=0.2\left((\Delta x)^{2}+(\Delta y)^{2}\right)$ are shown in Figure 3.15 for two different potentials.

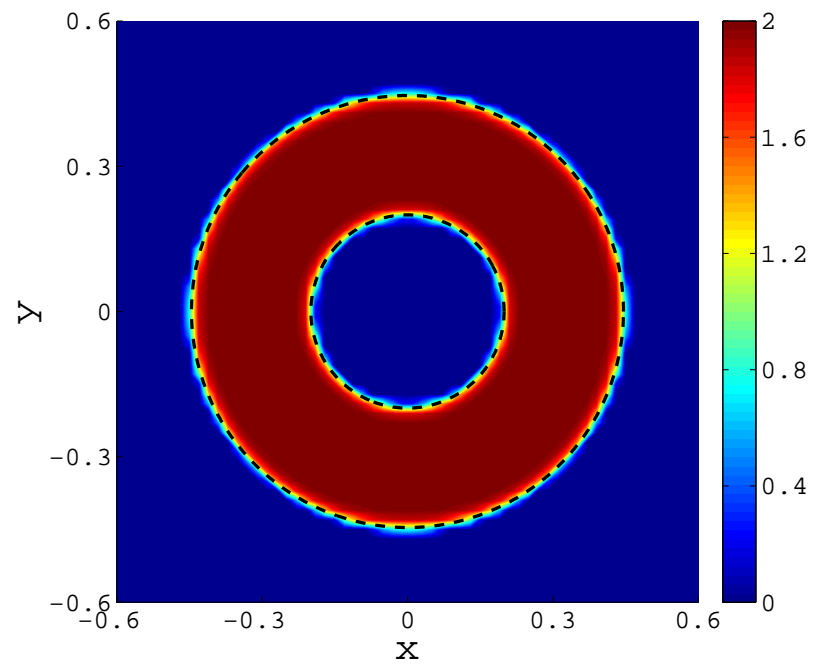

(a) $W(\mathbf{x})=|\mathbf{x}|^{2} / 2-\ln |\mathbf{x}|$

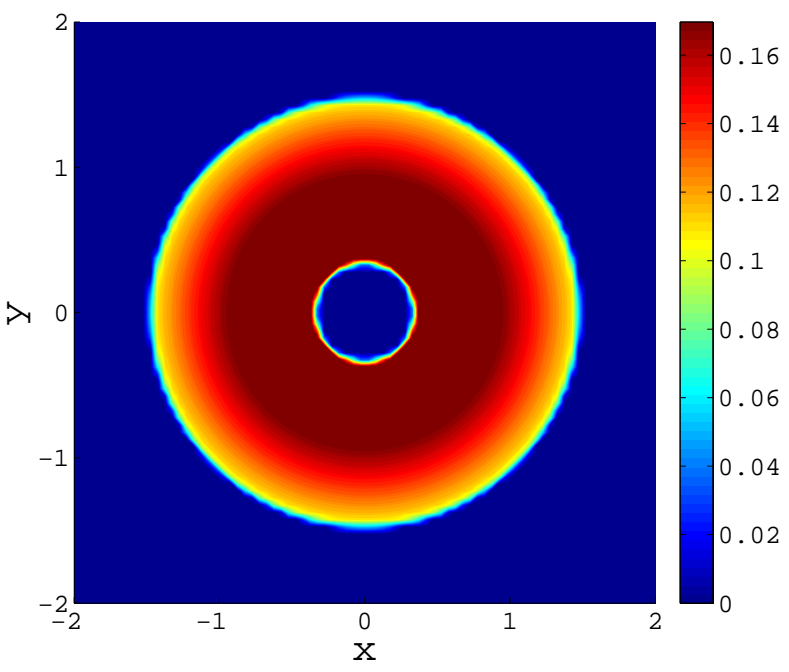

(b) $W(\mathbf{x})=\lambda(V(|\mathbf{x}|)-C V(|\mathbf{x}| / \ell))$

Figure 3.15: The steady density $\rho_{\infty}$ for the rotating mill with $\Delta x=\Delta y=0.05$. (a) $\alpha=0.25$, $\beta=2 \pi$; (b) $V(r)=-K_{0}(k r) / 2 \pi$, where $K_{0}(r)$ is the modified Bessel function of the second kind and the parameters $C=10 / 9, \ell=0.75, k=0.5, \lambda=100, \alpha=1.0, \beta=40$ are taken from [14].

Acknowledgment: JAC acknowledges support from projects MTM2011-27739-C04-02, 2009SGR-345 from Agència de Gestió d'Ajuts Universitaris i de Recerca-Generalitat de Catalunya, and the Royal Society through a Wolfson Research Merit Award. JAC and YH were supported by Engineering and Physical Sciences Research Council (UK) grant number EP/K008404/1. The work of AC was supported in part by the NSF Grant DMS-1115682. The authors also acknowledge the support by NSF RNMS grant DMS-1107444.

\section{References}

[1] L. Ambrosio, N. Gigli, And G. Savaré, Gradient flows in metric spaces and in the space of probability measures, Lectures in Mathematics ETH Zürich, Birkhäuser Verlag, Basel, second ed., 2008. 
[2] D. Balagué, J. A. Carrillo, T. Laurent, and G. Raoul, Dimensionality of local minimizers of the interaction energy, Arch. Ration. Mech. Anal., 209 (2013), pp. 10551088.

[3] D. Benedetto, E. Caglioti, J. A. Carrillo, and M. Pulvirenti, A nonMaxwellian steady distribution for one-dimensional granular media, J. Statist. Phys., 91 (1998), pp. 979-990.

[4] D. Benedetto, E. Caglioti, And M. Pulvirenti, A kinetic equation for granular media, RAIRO Modél. Math. Anal. Numér., 31 (1997), pp. 615-641.

[5] M. Bessemoulin-Chatard and F. Filbet, A finite volume scheme for nonlinear degenerate parabolic equations, SIAM J. Sci. Comput., 34 (2012), pp. B559-B583.

[6] A. Blanchet, E. A. Carlen, And J. A. Carrillo, Functional inequalities, thick tails and asymptotics for the critical mass Patlak-Keller-Segel model, J. Funct. Anal., 262 (2012), pp. 2142-2230.

[7] A. Blanchet, J. A. Carrillo, and P. Laurençot, Critical mass for a Patlak-KellerSegel model with degenerate diffusion in higher dimensions, Calc. Var. Partial Differential Equations, 35 (2009), pp. 133-168.

[8] A. Blanchet, J. A. Carrillo, and N. Masmoudi, Infinite time aggregation for the critical Patlak-Keller-Segel model in $\mathbb{R}^{2}$, Comm. Pure Appl. Math., 61 (2008), pp. 14491481.

[9] M. Burger, J. A. Carrillo, and M.-T. Wolfram, A mixed finite element method for nonlinear diffusion equations, Kinet. Relat. Models, 3 (2010), pp. 59-83.

[10] M. Burger, M. di Francesco, And M. Franek, Stationary states of quadratic diffusion equations with long-range attraction, Commun. Math. Sci., 11 (2013), pp. 709-738.

[11] M. Burger, R. Fetecau, And Y. Huang, Stationary states and asymptotic behaviour of aggregation models with nonlinear local repulsion, 2013.

[12] V. Calvez and J. A. Carrillo, Volume effects in the Keller-Segel model: energy estimates preventing blow-up, J. Math. Pures Appl. (9), 86 (2006), pp. 155-175.

[13] J. F. Campos and J. Dolbeault, Asymptotic estimates for the parabolic-elliptic kellersegel model in the plane, preprint, (2013).

[14] J. Carrillo, S. Martin, and V. Panferov, A new interaction potential for swarming models, Physica D: Nonlinear Phenomena, 260 (2013), pp. 112 - 126.

[15] J. A. Carrillo, M. R. D’Orsogna, and V. Panferov, Double milling in selfpropelled swarms from kinetic theory, Kinet. Relat. Models, 2 (2009), pp. 363-378.

[16] J. A. Carrillo, L. C. F. Ferreira, and J. C. Precioso, A mass-transportation approach to a one dimensional fluid mechanics model with nonlocal velocity, Adv. Math., 231 (2012), pp. 306-327. 
[17] J. A. Carrillo, R. J. MCCann, and C. Villani, Kinetic equilibration rates for granular media and related equations: entropy dissipation and mass transportation estimates, Rev. Mat. Iberoam., 19 (2003), pp. 971-1018.

[18] _ Contractions in the 2-Wasserstein length space and thermalization of granular media, Arch. Ration. Mech. Anal., 179 (2006), pp. 217-263.

[19] J. A. Carrillo And G. Toscani, Asymptotic $L^{1}$-decay of solutions of the porous medium equation to self-similarity, Indiana Univ. Math. J., 49 (2000), pp. 113-142.

[20] M. R. D’Orsogna, Y.-L. Chuang, A. L. Bertozzi, and L. S. Chayes, Self-propelled particles with soft-core interactions: patterns, stability, and collapse, Phys. Rev. Lett., 96 (2006), p. 104302.

[21] K. Fellner And G. Raoul, Stable stationary states of non-local interaction equations, Math. Models Methods Appl. Sci., 20 (2010), pp. 2267-2291.

[22] — Stability of stationary states of non-local equations with singular interaction potentials, Math. Comput. Modelling, 53 (2011), pp. 1436-1450.

[23] R. C. Fetecau, Y. Huang, and T. Kolokolnikov, Swarm dynamics and equilibria for a nonlocal aggregation model, Nonlinearity, 24 (2011), pp. 2681-2716.

[24] S. Gottlieb, C.-W. Shu, And E. TAdmor, Strong stability-preserving high-order time discretization methods, SIAM Rev., 43 (2001), pp. 89-112.

[25] E. F. Keller AND L. A. SEGEL, Initiation of slime mold aggregation viewed as an instability, Journal of Theoretical Biology, 26 (1970), pp. 399-415.

[26] H. Levine, W.-J. Rappel, And I. Cohen, Self-organization in systems of self-propelled particles, Phys. Rev. E, 63 (2000), p. 017101.

[27] H. Li And G. Toscani, Long-time asymptotics of kinetic models of granular flows, Arch. Ration. Mech. Anal., 172 (2004), pp. 407-428.

[28] K.-A. LIE AND S. NoELle, On the artificial compression method for second-order nonoscillatory central difference schemes for systems of conservation laws, SIAM J. Sci. Comput., 24 (2003), pp. 1157-1174.

[29] R. J. MCCAnn, A convexity principle for interacting gases, Adv. Math., 128 (1997), pp. $153-179$.

[30] H. Nessyahu And E. TADmor, Nonoscillatory central differencing for hyperbolic conservation laws, J. Comput. Phys., 87 (1990), pp. 408-463.

[31] F. Отто, The geometry of dissipative evolution equations: the porous medium equation, Comm. Partial Differential Equations, 26 (2001), pp. 101-174.

[32] E. B. SAFF AND V. Totik, Logarithmic potentials with external fields, vol. 316 of Grundlehren der Mathematischen Wissenschaften [Fundamental Principles of Mathematical Sciences], Springer-Verlag, Berlin, 1997. Appendix B by Thomas Bloom. 
[33] G. Ströhmer, Stationary states and moving planes, in Parabolic and Navier-Stokes equations. Part 2, vol. 81 of Banach Center Publ., Polish Acad. Sci. Inst. Math., Warsaw, 2008, pp. $501-513$.

[34] P. SWEBY, High resolution schemes using flux limiters for hyperbolic conservation laws, SIAM J. Numer. Anal., 21 (1984), pp. 995-1011.

[35] C. M. Topaz, A. L. Bertozzi, And M. A. Lewis, A nonlocal continuum model for biological aggregation, Bull. Math. Biol., 68 (2006), pp. 1601-1623.

[36] G. Toscani, One-dimensional kinetic models of granular flows, M2AN Math. Model. Numer. Anal., 34 (2000), pp. 1277-1291.

[37] B. VAN LEER, Towards the ultimate conservative difference scheme. V. A second-order sequel to Godunov's method, J. Comput. Phys., 32 (1979), pp. 101-136.

[38] J. L. VÁzQuez, The porous medium equation, Oxford Mathematical Monographs, The Clarendon Press Oxford University Press, Oxford, 2007. Mathematical theory.

[39] J. J. L. Velázquez, Point dynamics in a singular limit of the Keller-Segel model. I. Motion of the concentration regions, SIAM J. Appl. Math., 64 (2004), pp. 1198-1223.

[40] C. Villani, Topics in optimal transportation, vol. 58 of Graduate Studies in Mathematics, American Mathematical Society, Providence, RI, 2003.

[41] J. Von Zur Gathen And J. Gerhard, Modern computer algebra, Cambridge University Press, Cambridge, second ed., 2003.

[42] Y. YAO AND A. L. BertozzI, Blow-up dynamics for the aggregation equation with degenerate diffusion, Physica D: Nonlinear Phenomena, 260 (2013), pp. 77 - 89. 